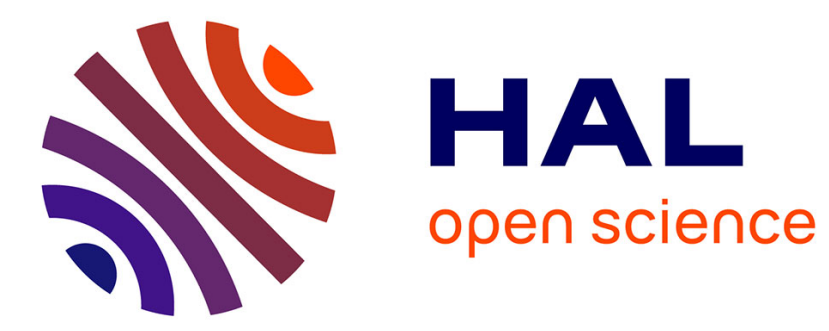

\title{
Boiling in porous media: Model and simulations
}

\author{
J. Bénard, Robert Eymard, Xavier Nicolas, C. Chavant
}

\section{To cite this version:}

J. Bénard, Robert Eymard, Xavier Nicolas, C. Chavant. Boiling in porous media: Model and simulations. Transport in Porous Media, 2005, 60 (1), pp.1-31. 10.1007/s11242-004-2594-9 . hal-00694587

\section{HAL Id: hal-00694587 \\ https://hal.science/hal-00694587}

Submitted on 4 May 2012

HAL is a multi-disciplinary open access archive for the deposit and dissemination of scientific research documents, whether they are published or not. The documents may come from teaching and research institutions in France or abroad, or from public or private research centers.
L'archive ouverte pluridisciplinaire HAL, est destinée au dépôt et à la diffusion de documents scientifiques de niveau recherche, publiés ou non, émanant des établissements d'enseignement et de recherche français ou étrangers, des laboratoires publics ou privés. 


\title{
Boiling in porous media. Model and simulations.
}

\author{
Johann Bénard, Robert Eymard and Xavier Nicolas
}

Laboratoire d'Étude des Transferts d'Énergie et de Matière

Université de Marne la Vallée - Bât. Lavoisier - 77454 Champs sur Marne - France

\section{Clément Chavant}

EDF RED, Département Analyses Mécaniques et Acoustiques

1, av du Général de Gaulle - 92141 Clamart Cedex - France

April 2, 2004

\begin{abstract}
We present a modelization of the heat and mass transfers within a porous medium, which takes into account phase transitions. Classical equations are derived for the mass conservation equation, whereas the equation of energy relies on an entropy balance adapted to the case of a rigid porous medium. The approximation of the solution is obtained using a finite volume scheme coupled with the management of phase transitions. This model is shown to apply in the case of an experiment of heat generation in a porous medium. The vapor phase appearance is well reproduced by the simulations, and the size of the two-phase region is correctly predicted. A result of this study is the evidence of the discrepancy between the air - water capillary and relative permeability curves and the water - water vapor ones.
\end{abstract}

Keywords: two-phase flow in porous media, phase transition, finite volume method, water - water vapor capillary curve.

\section{Nomenclature:}

(C) 2004 Kluwer Academic Publishers. Printed in the Netherlands. 
$\mathcal{A}_{i j} \quad$ Measure of interface between grid blocks $i$ and $j, m^{2}$

$B \quad$ Equation and inequality associated with the thermodynamic state

$C_{p} \quad$ Mass heat capacity of phase $p, J \cdot \mathrm{kg}^{-1} \cdot K^{-1}$

$D \quad$ Discrete system of equations

$d_{i j} \quad$ Distance between centers of control volumes $i$ and $j, m$

E Internal energy per volume unit, $J . m^{-3}$

$F_{w} \quad$ Mass flux of water, $\mathrm{kg} \cdot \mathrm{s}^{1}$

$F_{h} \quad$ Heat flux, $W$

$g_{p} \quad$ Gibbs potential of phase $p$ per mass unit, $J . \mathrm{kg}^{-1}$

$h_{p} \quad$ Enthalpy of phase $p$ per mass unit, $J . k g^{-1}$

$K \quad$ Absolute permeability, $m^{2}$

$k_{r p} \quad$ Relative permeability of phase $p$

$m_{p} \quad$ Mass of phase $p$ per porous volume unit, $\mathrm{kg} \cdot \mathrm{m}^{-3}$

$M_{w} \quad$ Molar weight of water, $k g \cdot m o l e^{-1}$

$N_{c v} \quad$ Number of control volumes

$P_{p} \quad$ Pressure of phase $p, P a$

$P_{c} \quad$ Capillary pressure, $\mathrm{Pa}$

q Conductive thermal flux, W. $m^{-2}$

$\bar{Q} \quad$ Heat source term, W. $m^{-3}$

$\bar{Q}_{i} \quad$ Heat source term, $W$

$S \quad$ Liquid saturation

$T \quad$ Temperature, $K$

$t \quad$ Time, $s$

$\mathcal{U} \quad$ Discrete unknowns

$\mathcal{V}_{i} \quad$ Measure of grid blocks $i, m^{3}$

$\mathbf{V}_{\mathbf{p}} \quad$ Volumic flux of phase $p, m^{3} \cdot s^{-1}$

$\mathbf{v}_{\mathbf{p}} \quad$ Specific flux of phase $p, m . s^{-1}$

$x, y, z \quad$ Space coordinates, $m$ 
Greek symbols

$\eta \quad$ Entropy per mass unit, $J . K^{-1} \cdot \mathrm{kg}^{-1}$

$\tilde{\eta} \quad$ Entropy per volume unit, $J . K^{-1} \cdot m^{-3}$

$\lambda \quad$ Thermal conductivity, $W \cdot m^{-1} \cdot K^{-1}$

$\lambda_{\text {wet }}$ Thermal conductivity of the saturated porous medium, $W \cdot m^{-1} \cdot K^{-1}$

$\lambda_{\text {dry }}$ Thermal conductivity of the dry porous medium, $W \cdot m^{-1} \cdot K^{-1}$

$\mu_{p} \quad$ Dynamic viscosity of phase $p, P a . s$

$\rho_{p} \quad$ Bulk density of phase $p, k g \cdot m^{-3}$

$\tau_{l \rightarrow v}^{w} \quad$ Mass rate of water transfer from phase $l$ to phase $v$ per volume unit, $\mathrm{kg} \cdot \mathrm{m}^{-3} \cdot \mathrm{s}^{-1}$

$\phi \quad$ Porosity

$\varphi \quad$ Dissipations

$\chi \quad$ Indicator of thermodynamic state of grid block $i$ $(\chi=1,2,3)$

Subscripts and superscripts

c Capillarity

$h \quad$ Heat

$l \quad$ Liquid

$p \quad$ Phase

$s \quad$ Solid

$v \quad$ Vapor

$w \quad$ Water

$w-a$ Relative to the couple water and air

$w-v \quad$ Relative to the couple water and water vapor 


\section{Introduction}

Heat transfer and fluid flow with liquid-vapor phase transition in porous media arise in a number of scientific and engineering disciplines. Important technological applications can be found in various domain. The mechanical behavior of drying porous materials must be known in civil engineering applications (Whitaker, 1998; Coussy et al, 1998). In petroleum engineering, multiple flowing phases are present in natural oil reservoirs and various enhanced multi-phase exploitation techniques, such as water and vapor flooding, are employed (Woods, 1999). For the purpose of the nuclear reactor safety analysis, the understanding of flow and transport mechanism of vapor through the concrete enclosure is essential (Medhekar et al, 1991). The study of the storage or disposal of nuclear waste strongly involves the prediction of the long-term heating of porous media due to the residual radioactivity. Since, in this last technical area, it is particularly difficult to manage accurate experiments for long-term storage, it has been undertaken to establish the main physical and chemical mechanisms that govern the behavior of waste packages disposals (Toulhoat, 2002). The prediction of thresholds for acceptable heating, in nominal or accidental operating conditions, depends on models validated under the actual disposal conditions (Castelier, 2001).

Here, we study some features of this problem, focusing on the modelization and the numerical simulation of heat and mass transfers in initially saturated porous media, taking into account phase transition phenomena. 
In this paper, our approach for treating this problem consists in considering the various liquid phases as distinct fluids with individual thermodynamic and transport properties and with different phase specific fluxes. The transport phenomena are then mathematically described by the basic balance equations for each phase separately. In this direction, (Ramseh et al, 1993) propose a model where interfaces separating single from two phase regions are tracked, whereas (Daurelle et al, 1998) propose a model where the liquid and the gaseous phases coexist in any point. The model that we present here includes the determination of the thermodynamic equilibrium state at each point of the porous medium, and the appearance or the disappearance of phases. This model, which is an extension of (Wang et al, 1993; Ghafir, 2000; Najjari et al, 2002) to cases where the energy equation cannot be written as an enthalpy balance, can be described by a set of equations, coupled with inequalities.

The outline of the article is as follows. In Section 2, we describe the continuous equations of the model and the management of the phase transition. Then, in Section 3, we outline the numerical methods used to find an approximate solution to the system of equations (in an appendix, a comparison between numerical and analytical solutions, in some simplified cases, provides a validation of these methods). In Section 4, we proceed to comparison between experimental results and computational ones. A method of determining the vapor capillary pressure and a parametric study of the water vapor relative permeability is detailed in Section 5. Some conclusions and future works are then drawn in Section 6. 


\section{Description of the model}

\subsection{MASS CONSERVATION AND ENERGy EQUATIONS}

The porous medium is treated as a unique continuous medium resulting from the super-imposition of the skeleton and fluid continua. The skeleton continuum is rigid and the fluid continuum is composed by two fluids (liquid water and water vapor), assuming that there is no dry air. We assume the existence of a representative elementary volume which is relevant at the macroscopic scale for all the physical phenomena involved in the intended application. Moreover, at any point of the continuous medium, the three phases are locally at thermal equilibrium $\left(T_{s}=T_{l}=T_{v}=T\right)$. Under these conditions, we use the following classical equations to model the flows in the porous medium. The liquid phase conservation as well as the vapor phase conservation are expressed by:

$$
\left\{\begin{array}{l}
\frac{\partial m_{l}}{\partial t}+\nabla \cdot\left(\rho_{l} \mathbf{v}_{\mathbf{l}}\right)=-\tau_{l \rightarrow v}^{w} \\
\frac{\partial m_{v}}{\partial t}+\nabla \cdot\left(\rho_{v} \mathbf{v}_{\mathbf{v}}\right)=+\tau_{l \rightarrow v}^{w}
\end{array}\right.
$$

where

$$
m_{l}=\phi S \rho_{l} \quad \text { and } \quad m_{v}=\phi(1-S) \rho_{v} .
$$

In the above equations, $\phi$ is the porosity of porous medium supposed to be a constant, $S$ is the liquid saturation, $\rho_{p}\left(P_{p}, T\right)$ is the density of phase $p=l, v$, state function of the pressure $P_{p}$ of phase $p$ and of the temperature $T$. We denote by $\mathbf{v}_{\mathbf{p}}$ the specific flux of the phase 
$p=l$ or $v$. The term $\tau_{l \rightarrow v}^{w}$ represents the mass rate per volume unit transfered from the liquid to the vapor phase.

Following Coussy $(1995,2004)$ for the formulation of the energy equation in the case of an open volume unit of a rigid porous medium, under quasi statics evolutions assumptions, the first law of thermodynamics produces

$$
\frac{\partial E}{\partial t}=-\nabla \cdot \sum_{p=l, v} h_{p} \rho_{p} \mathbf{v}_{\mathbf{p}}-\nabla \cdot \mathbf{q}+\mathbf{g} \cdot \sum_{p=l, v} \rho_{p} \mathbf{v}_{\mathbf{p}}+\bar{Q}
$$

where $E, h_{p}\left(P_{p}, T\right), \mathbf{q}, \mathbf{g}$ and $\bar{Q}$ are respectively the internal energy per volume unit, the enthalpy per mass unit of phase $p$, the conductive heat flux, the gravity acceleration and the heat source terms. The second law of thermodynamics can be expressed by

$$
\frac{\partial \tilde{\eta}}{\partial t} \geq-\nabla \cdot\left(\sum_{p=l, v} \eta_{p} \rho_{p} \mathbf{v}_{\mathbf{p}}+\frac{\mathbf{q}}{T}\right)+\frac{\bar{Q}}{T}
$$

with

$$
\tilde{\eta}=\phi S \rho_{l} \eta_{l}+\phi(1-S) \rho_{v} \eta_{v}+(1-\phi) \rho_{s} \eta_{s}
$$

In the above relations, we denote by $\eta_{p}\left(P_{p}, T\right)$ the entropy per mass unit of the liquid and vapor phases $(p=l$ and $v)$ and we denote by $\eta_{s}(T)=C_{s} \log \left(T / T_{0}\right)$ the entropy per mass unit of the solid phase. We suppose that $\rho_{s}$ is constant. The expression of $-\nabla \cdot \mathbf{q}+\bar{Q}$, obtained from (3), is introduced in (4) multiplied by $T$. Thanks to the expression $g_{p}\left(P_{p}, T\right)=h_{p}\left(P_{p}, T\right)-T \eta_{p}\left(P_{p}, T\right)$ of the Gibbs potential per mass unit of phase $p=l, v$, we get

$$
\varphi_{\mathrm{int}}+\varphi_{\text {flow }}+\varphi_{\text {therm }} \geq 0
$$


where the term $\varphi_{\text {int }}$, representing the intrinsic dissipation, is given by

$$
\varphi_{\mathrm{int}}=T \frac{\partial \tilde{\eta}}{\partial t}-\frac{\partial E}{\partial t}-\sum_{p=l, v} g_{p} \nabla \cdot\left(\rho_{p} \mathbf{v}_{\mathbf{p}}\right)
$$

the term $\varphi_{\text {flow }}$, representing the dissipation due to the mass transfer, is given by

$$
\varphi_{\text {flow }}=-\sum_{p=l, v} \rho_{p} \mathbf{v}_{\mathbf{p}} \cdot\left(\nabla g_{p}+\eta_{p} \nabla T-\mathbf{g}\right)=-\sum_{p=l, v} \rho_{p} \mathbf{v}_{\mathbf{p}} \cdot\left(\frac{1}{\rho_{p}} \nabla P_{p}-\mathbf{g}\right)
$$

and the term $\varphi_{\text {therm }}$, representing the dissipation due to the heat transfer, is given by

$$
\varphi_{\text {therm }}=-\frac{\mathbf{q}}{T} \cdot \nabla T
$$

Since the porous medium is assumed to be rigid, the intrinsic dissipation $\varphi_{\text {int }}$ is equal to zero. This gives, thanks to Equation (3)

$$
T \frac{\partial \tilde{\eta}}{\partial t}-\sum_{p=l, v} g_{p} \nabla \cdot \rho_{p} \mathbf{v}_{\mathbf{p}}=-\nabla \cdot\left(\sum_{p=l, v} h_{p} \rho_{p} \mathbf{v}_{\mathbf{p}}+\mathbf{q}\right)+g \cdot \sum_{p=l, v} \rho_{p} \mathbf{v}_{\mathbf{p}}+\bar{Q}
$$

Using mass conservation Equations (1), we get

$$
\begin{aligned}
T \frac{\partial \tilde{\eta}}{\partial t}+\sum_{p=l, v} g_{p} \frac{\partial m_{p}}{\partial t}+\tau_{l \rightarrow v}^{w}\left(g_{l}-g_{v}\right)= & -\nabla \cdot \sum_{p=l, v} h_{p} \rho_{p} \mathbf{v}_{\mathbf{p}}+\mathbf{q} \\
& +g \cdot \sum_{p=l, v} \rho_{p} \mathbf{v}_{\mathbf{p}}+\bar{Q}
\end{aligned}
$$

Assuming that there is no dissipation due to the phase transition, i.e.

$$
\tau_{l \rightarrow v}^{w}\left(g_{l}-g_{v}\right)=0
$$


and neglecting the mechanical energy due to the volumic weight forces, we get:

$$
T \frac{d \tilde{\eta}}{d t}+\sum_{p=l, v} g_{p} \frac{d m_{p}}{d t}=-\nabla \cdot\left(\sum_{p=l, v} h_{p} \rho_{p} \mathbf{v}_{\mathbf{p}}\right)-\nabla \cdot \mathbf{q}+\bar{Q}
$$

We satisfy the condition $\varphi_{\text {therm }} \geq 0$, assuming that the conductive heat flux is given by Fourier's law, in which we use an effective conductivity taking into account the water content of the porous medium:

$$
\mathbf{q}=-\lambda(S) \nabla T
$$

Different expressions of $\lambda(S)$ are available in the literature (see for example (De Vries, 1964; Kelly et al, 1983)). Note that the influence of a given law is essentially governed by the values $\lambda(0)=\lambda_{\text {dry }}$ and $\lambda(1)=\lambda_{\text {wet }}$, since for $0<S<1$, the temperature is determined by the equilibrium between water and water vapor, leading to small gradient in the two-phase zone. Nevertheless, following (Wang et al, 1993), we use a linear effective thermal conductivity law $\lambda(S)=S \lambda_{\text {wet }}+(1-S) \lambda_{\text {dry }}$.

Finally, we satisfy the condition $\varphi_{\text {flow }} \geq 0$, assuming that the velocity of phase $p$ is given by Darcy's law:

$$
\mathbf{v}_{\mathbf{p}}=\frac{k_{r p} K}{\mu_{p}}\left(-\nabla P_{p}+\rho_{p} \mathbf{g}\right),
$$

where $K$ is the absolute permeability of the porous medium (assumed here to be constant), $k_{r p}(S)$, the relative permeability of phase $p$, is a function of the liquid saturation $\left(k_{r l}(S)\right.$ is an increasing function such that $k_{r l}(0)=0$ and $k_{r v}(S)$ is a decreasing function such that $\left.k_{r v}(1)=0\right)$ and $\mu_{p}(T)$ is the dynamic viscosity of phase $p$, assumed 
to only depend on the temperature. Using Equations (1), (2) and (10), the mass conservation equation writes:

$$
\frac{\partial}{\partial t}\left(\phi S \rho_{l}+\phi(1-S) \rho_{v}\right)+\nabla \cdot\left[\begin{array}{l}
\rho_{l} \frac{k_{l l} K}{\mu_{l}}\left(-\nabla P_{l}+\rho_{l} \mathbf{g}\right) \\
+\rho_{v} \frac{k_{r_{v}} K}{\mu_{v}}\left(-\nabla P_{v}+\rho_{v} \mathbf{g}\right)
\end{array}\right]=0
$$

Using Equations (1), (2), (8), (9) and (10), the energy equation is expressed by

$$
\begin{aligned}
& {\left[\begin{array}{l}
T \frac{\partial}{\partial t}\left(\phi S \rho_{l} \eta_{l}+\phi(1-S) \rho_{v} \eta_{v}\right) \\
+T \frac{\partial}{\partial t}\left((1-\phi) \rho_{s} \eta_{s}\right)+ \\
g_{l} \frac{\partial}{\partial t}\left(\phi S \rho_{l}\right)+g_{v} \frac{\partial}{\partial t}\left(\phi(1-S) \rho_{v}\right)
\end{array}\right]+\nabla \cdot\left[\begin{array}{l}
h_{l} \rho_{l} \frac{k_{r l} K}{\mu_{l}}\left(-\nabla P_{l}+\rho_{l} \mathbf{g}\right) \\
+h_{v} \rho_{v} \frac{k_{r v} K}{\mu_{v}}\left(-\nabla P_{v}+\rho_{v} \mathbf{g}\right) \\
-\left(\lambda_{\text {wet }} S+\lambda_{\text {dry }}(1-S)\right) \nabla T
\end{array}\right]} \\
& =\bar{Q} .
\end{aligned}
$$

The vapor pressure $P_{v}$ is related to the liquid pressure using the capillary pressure, which is a decreasing function of the liquid saturation:

$$
P_{c}(S)=P_{v}-P_{l}
$$

Equation (7) is not sufficient to close system (11), (12), (13) with respect to $\left(P_{l}, P_{v}, S, T\right)$. We therefore give in the next section sufficient conditions, which ensure (7), and which enable to calculate the thermodynamic state at each point.

\subsection{CONDitions For the PHASE TRANSition}

The equilibrium thermodynamic state of the water (one phase liquid, one phase vapor, or two-phase) can be determined for given liquid and gaseous pressures and temperature conditions, using the computation 
of the Gibbs potential for each phase. When Gibbs potentials are equal, both phases are in equilibrium; otherwise, the phase with the maximum Gibbs potential disappears to the benefit of the phase with the minimum Gibbs potential. Therefore, three equilibrium states are possible:

State $1:$ no vapor phase, $S=1$ and $g_{l}\left(P_{l}, T\right)<g_{v}\left(P_{v}, T\right)$

State 2 : liquid-vapor equilibrium, $g_{l}\left(P_{l}, T\right)=g_{v}\left(P_{v}, T\right)$

and $0<S<1$

State $3:$ no liquid phase, $S=0$ and $g_{l}\left(P_{l}, T\right)>g_{v}\left(P_{v}, T\right)$

Note that, in State 1 , we get $m_{v}=0$ and $\mathbf{v}_{\mathbf{v}}=0$, which delivers, using (1), $\tau_{l \rightarrow v}^{w}=0$. In State 3, we then have $m_{l}=0$ and $\mathbf{v}_{\mathbf{l}}=0$, and the same conclusion holds. Therefore, equation (7) is satisfied.

System (11), (13), (8) and (14) is now closed, with respect to the four unknowns $P_{l}, P_{v}, S$ and $T$.

\subsection{State FUnCtions FOR The LiQUid AND VAPOR WATER PHASES}

In this model, we need the expressions of the density, the dynamic viscosity, the enthalpy and the entropy of each water phase $p=l, v$ as explicit state functions of the pressure of the phase and of the common temperature. We assume that for $p=l, v$, the mass heat capacity $\frac{\partial h_{p}}{\partial T}\left(P_{p}, T\right)$ does not depend on the pressure $P_{p}$, and therefore verifies $\frac{\partial h_{p}}{\partial T}\left(P_{p}, T\right)=C_{p}(T)$. By integration, introducing a reference state (specified below) defined by the pressure $P_{0}$ and the temperature $T_{0}$, and a constant $h_{p 0}$, we get the existence of a function $\xi\left(P_{p}\right)=$ 
$\frac{\partial h_{p}}{\partial P_{p}}\left(P_{p}, T\right)$ such that

$$
h_{p}\left(P_{p}, T\right)=h_{p 0}+\int_{T_{0}}^{T} C_{p}(\tau) d \tau+\int_{P_{0}}^{P_{p}} \xi(\pi) d \pi
$$

Since we have

$$
d h_{p}\left(P_{p}, \eta_{p}\right)=T d \eta_{p}+\frac{1}{\rho_{p}} d P_{p}
$$

we get that

$$
d \eta_{p}\left(P_{p}, T\right)=\frac{C_{p}(T)}{T} d T-\frac{1}{T}\left(\frac{1}{\rho_{p}}-\xi\left(P_{p}\right)\right) d P_{p}
$$

This implies using Maxwell relations that:

$$
\frac{\partial}{\partial T} \frac{\frac{1}{\rho_{p}}-\xi\left(P_{p}\right)}{T}=0
$$

which gives the existence of a function $\zeta\left(P_{p}\right)$ such that

$$
\frac{\frac{1}{\rho_{p}}-\xi\left(P_{p}\right)}{T}=\zeta\left(P_{p}\right)
$$

We thus deduce the following expression for the density of the phase:

$$
\rho_{p}\left(P_{p}, T\right)=\frac{1}{T \zeta\left(P_{p}\right)+\xi\left(P_{p}\right)}
$$

We then obtain that

$$
d \eta_{p}\left(P_{p}, T\right)=\frac{C_{p}(T)}{T} d T-\zeta\left(P_{p}\right) d P
$$

and therefore, there exists an integration constant $\eta_{p 0}$ such that

$$
\eta_{p}\left(P_{p}, T\right)=\eta_{p 0}+\int_{T_{0}}^{T} \frac{C_{p}(\tau)}{\tau} d \tau-\int_{P_{0}}^{P_{p}} \zeta(\pi) d \pi
$$


Relations (15-17) then provide consistent thermodynamic functions for the class of materials whose mass heat capacity only depends on the temperature. For the sake of simplicity, we consider a constant density for the liquid water. This corresponds to the choice $\zeta_{l}\left(P_{l}\right)=0$ and $\xi_{l}\left(P_{l}\right)=1 / \rho_{l 0}$, with $\rho_{l 0}=957.9 \mathrm{~kg} \cdot \mathrm{m}^{-3}$, and we set $C_{l}(T)=C_{l 0}=$ $4196 \mathrm{~J}_{\mathrm{kg}} \mathrm{g}^{-1} \cdot \mathrm{K}^{-1}$. Note that it is possible to increase the accurateness of these functions, setting $\zeta_{l}\left(P_{l}\right)=a / \rho_{l 0}$ and $\xi_{l}\left(P_{l}\right)=\left(1-b\left(P_{l}-\right.\right.$ $\left.\left.P_{0}\right)-a T_{0}\right) / \rho_{l 0}$. It then suffices to select $a$ and $b$ with respect to the compressibility and the dilatability of liquid water in the considered range of temperature and pressure.

Assuming the water vapor to be an ideal gas, we write $\zeta_{v}\left(P_{v}\right)=$ $\frac{R}{M_{w} P_{v}}$, with $R=8.315 \mathrm{~J} . K^{-1} \cdot \mathrm{mole} e^{-1}$ and $M_{w}=1810^{-3} \mathrm{~kg} \cdot \mathrm{mole}^{-1}$, $\xi_{v}\left(P_{v}\right)=0$, and we set $C_{v}(T)=C_{v 0}=1870 \mathrm{~J} . \mathrm{kg}^{-1} \cdot \mathrm{K}^{-1}$.

The four constants $h_{l 0}, \eta_{l 0}, h_{v 0}$ and $\eta_{v 0}$ cannot be chosen independently. Indeed, considering the reference equilibrium state at the atmospheric pressure $P_{0}=1.0132510^{5} \mathrm{~Pa}, T_{0}=373 \mathrm{~K}$, we must ensure $g_{v}\left(P_{0}, T_{0}\right)=g_{l}\left(P_{0}, T_{0}\right)$ and $\eta_{v}\left(P_{0}, T_{0}\right)-\eta_{l}\left(P_{0}, T_{0}\right)=L_{0} / T_{0}$, where the latent heat $L_{0}$ at this reference state is equal to $L_{0}=$ $225710^{3} \mathrm{~J}_{\mathrm{kg}} \mathrm{kg}^{-1}$. We can therefore take $h_{l 0}=0, \eta_{l 0}=0, h_{v 0}=L_{0}$, $\eta_{v 0}=L_{0} / T_{0}$. Gathering the previous expressions, we obtain the table I. Using the expressions given by table I, it can be verified that the equilibrium pressure function $P(T)$ such that $g_{v}(P(T), T)=g_{l}(P(T), T)$ and the equilibrium latent heat $L(T)=T\left(\eta_{v}(P(T), T)-\eta_{l}(P(T), T)\right)$ are close to that which can be found in the literature (Rohsenow et al, 1998 ) in the concerned range of temperatures and pressures (see figure 1). We remark that the above expressions of the phase densities are sufficient to ensure Kelvin's law, that is, for two equilibrium states $P_{l}$, 
Table I. Thermo-physical properties of water

\begin{tabular}{|c|c|c|c|}
\hline & notation & value & units \\
\hline Reference pressure & $P_{0}$ & $1.0132510^{5}$ & $\mathrm{~Pa}$ \\
\hline Reference temperature & $T_{0}$ & 373 & $K$ \\
\hline Liquid heat capacity & $C_{l 0}$ & 4196 & $J . k g^{-1} \cdot K^{-1}$ \\
\hline Vapor heat capacity & $C_{v 0}$ & 1870 & $J . k g^{-1} \cdot K^{-1}$ \\
\hline Latent heat at $T_{0}, P_{0}$ & $L_{0}$ & $225710^{3}$ & $J . k g^{-1}$ \\
\hline Gas constant for water vapor & $R$ & 8.315 & $J . K^{-1} \cdot$ mole $^{-1}$ \\
\hline Molar weight of water & $M_{w}$ & $1810^{-3}$ & $k g . m o l e^{-1}$ \\
\hline Liquid bulk density & $\rho_{l}\left(P_{l}, T\right)$ & $\rho_{l 0}=957.9$ & $\mathrm{~kg} \cdot \mathrm{m}^{-3}$ \\
\hline Liquid viscosity & $\mu_{l}(T)$ & {$\left[\begin{array}{l}10^{-3} \exp \left(\frac{4.20910^{3}}{\mathrm{~T}}-24.71\right. \\
\left.+4.52710^{-2} T-3.37610^{-5} T^{2}\right)\end{array}\right.$} & Pa.s \\
\hline Liquid enthalpy & $h_{l}\left(P_{l}, T\right)$ & $C_{l 0}\left(T-T_{0}\right)+\frac{1}{\rho_{l 0}}\left(P_{l}-P_{0}\right)$ & $J . k g^{-1}$ \\
\hline Liquid entropy & $\eta_{l}\left(P_{l}, T\right)$ & $C_{l 0} \log \left(T / T_{0}\right)$ & $J . k g^{-1} \cdot K^{-1}$ \\
\hline Liquid Gibbs potential & $g_{l}\left(P_{l}, T\right)$ & $h_{l}\left(P_{l}, T\right)-T \eta_{l}\left(P_{l}, T\right)$ & $J . k g^{-1}$ \\
\hline Vapor bulk density & $\rho_{v}\left(P_{v}, T\right)$ & $R T / M_{w} P_{v}$ & $k g \cdot m^{-3}$ \\
\hline Vapor viscosity & $\mu_{v}$ & $1.2710^{-5}$ & Pa.s \\
\hline Vapor enthalpy & $h_{v}\left(P_{v}, T\right)$ & $L_{0}+C_{v 0}\left(T-T_{0}\right)$ & $J . k g^{-1}$ \\
\hline Vapor entropy & $\eta_{v}\left(P_{v}, T\right)$ & {$\left[\begin{array}{l}L_{0} / T_{0}+C_{v 0} \log \left(T / T_{0}\right) \\
-\frac{R}{M_{w}} \log \left(P_{v} / P_{0}\right)\end{array}\right]$} & $J . k g^{-1} \cdot K^{-1}$ \\
\hline Vapor Gibbs potential & $g_{v}\left(P_{v}, T\right)$ & $h_{v}\left(P_{v}, T\right)-T \eta_{v}\left(P_{v}, T\right)$ & $J . k g^{-1}$ \\
\hline
\end{tabular}

$P_{v}, T$ and $\bar{P}_{l}, \bar{P}_{v}, T$

$$
\log \frac{P_{v}}{\bar{P}_{v}}=\frac{M_{w}}{\rho_{l 0} R T}\left(P_{l}-\bar{P}_{l}\right)
$$

Indeed, the above equation is immediately obtained by subtracting the two equations $g_{l}\left(P_{l}, T\right)=g_{v}\left(P_{v}, T\right)$ and $g_{l}\left(\bar{P}_{l}, T\right)=g_{v}\left(\bar{P}_{v}, T\right)$.

\section{Numerical techniques}

It is now necessary, in order to validate this model, to obtain a numerical approximation of the solution. This is completed using a finite 
volume method, which consists in a set of nonlinear discrete balance equations in grid blocks, coupled with the set of equations and inequalities resulting from the phase transition determination. This system is solved using Newton's method, the thermodynamic state of each grid block being updated at each iteration of the method. The advantage in this case of a finite volume method over other methods is that there is no need to assume a minimum value for the gas phase saturation (for example, some finite element methods require that both phases are mobile everywhere in order to ensure the invertibility of the rigidity matrix (Schrefler et al, 1993)). Thus, it is neither necessary to assume the presence of both phases everywhere nor to track the interface between the liquid one-phase region and the two-phase one. We consider a finite volume mesh of the domain, consisting of $N_{c v}$ grid blocks indexed by $i$ $\left(i=1, \ldots, N_{c v}\right)$. We denote by $\mathcal{V}_{i}$ the volume of the cell $i$, the subscript $j$ stands for any cell having a common interface $i \mid j$ with the cell $i, \mathcal{A}_{i j}$ is the measure of interface $i \mid j, d_{i j}$ is the distance between the centers of grid blocks $i$ and $j$.

Equations (11) and (8) give, after time discretization and finite volume discretization, a set of coupled nonlinear equations (Eymard et al, 2000):

$$
\left\{\begin{array}{l}
\mathcal{V}_{i} \frac{m_{w i}^{n+1}-m_{w i}^{n}}{t^{n+1}-t^{n}}+\sum_{i \mid j} F_{w i j}^{n+1}=0 \\
\mathcal{V}_{i}\left[T_{i}^{n+1} \frac{\tilde{\eta}_{h i}^{n+1}-\tilde{\eta}_{h i}^{n}}{t^{n+1}-t^{n}}+\sum_{p=l, v} g_{p i}^{n+1} \frac{m_{p i}^{n+1}-m_{p i}^{n}}{t^{n+1}-t^{n}}\right]+\sum_{i \mid j} F_{h i j}^{n+1}=\bar{Q}_{i}^{n+1}
\end{array}\right.
$$

In the above set of equations, we denote by $m_{w}=m_{l}+m_{v}$ the water mass and by $\bar{Q}_{i}^{n+1}$ the heat source term in grid block $i$. The water flux 
$F_{w i j}^{n+1}$ and the energy flux $F_{h i j}^{n+1}$ across the interface $i \mid j$ are evaluated using the following implicit finite difference scheme with respect to the pressures, the saturations and the temperatures of grid blocks $i$ and $j$ :

$$
\begin{gathered}
F_{w i j}^{n+1}=\rho_{l i j}^{n+1} V_{l i j}^{n+1}+\rho_{v i j}^{n+1} V_{v i j}^{n+1} \\
F_{h i j}^{n+1}=\left(\rho_{l} h_{l}\right)_{i j}^{n+1} V_{l i j}^{n+1}+\left(\rho_{v} h_{v}\right)_{i j}^{n+1} V_{v i j}^{n+1} \\
-\mathcal{A}_{i j} \frac{2 \lambda_{i}^{n+1} \lambda_{j}^{n+1}}{\lambda_{i}^{n+1}+\lambda_{j}^{n+1}} \frac{T_{i}^{n+1}-T_{j}^{n+1}}{d_{i j}}
\end{gathered}
$$

with (for $p=l, v)$

$$
V_{p i j}^{n+1}=\mathcal{A}_{i j} \frac{K k_{r p}\left(S_{p i j}^{n+1}\right)}{\mu_{p}}\left[\frac{P_{p i}^{n+1}-P_{p j}^{n+1}+\rho_{p i}^{n} g\left(z_{i}^{n+1}-z_{j}^{n+1}\right)}{d_{i j}}\right](2
$$

eliminating the vapor pressure using $P_{v i}^{n+1}=P_{l i}^{n+1}+P_{c}\left(S_{i}^{n+1}\right)$, and setting $\lambda_{i}^{n+1}=\lambda_{\text {wet }} S_{i}^{n+1}+\lambda_{\text {dry }}\left(1-S_{i}^{n+1}\right)$. In $(19,20,21)$ the density $\rho_{i j}$ and the liquid saturation $S_{p i j}$ at interface are estimated thanks to a phase by phase upwind scheme:

$$
S_{p i j}^{n+1}= \begin{cases}S_{j}^{n+1} & \text { if } P_{p i}^{n+1}-P_{p j}^{n+1}+\rho_{p i}^{n} g\left(z_{i}^{n+1}-z_{j}^{n+1}\right) \geq 0 \\ S_{i}^{n+1} & \text { if not. }\end{cases}
$$

Setting, for each cell $i, \chi_{i}^{n+1}$ the thermodynamic state of the cell $i$ (1 for pure liquid, 2 for liquid-vapor equilibrium, 3 for pure vapor), $\mathcal{U}_{i}^{n+1}=\left(P_{l i}^{n+1}, S_{i}^{n+1}, T_{i}^{n+1}\right)$ the triplet of unknowns of the cell $i$ (liquid pressure, liquid saturation, temperature) and

$$
\begin{aligned}
& B\left(\mathcal{U}_{i}^{n+1}, 1\right)=S-1 \\
& B\left(\mathcal{U}_{i}^{n+1}, 2\right)=g_{l}\left(P_{l}, T\right)-g_{v}\left(P_{v}, T\right) \\
& B\left(\mathcal{U}_{i}^{n+1}, 3\right)=S
\end{aligned}
$$


the numerical implementation of the equilibrium equations and inequalities (14) given in Subsection 2.2 is the following:

$$
\begin{aligned}
& \text { If } \chi_{i}^{n+1}=1 \text {, then } B\left(\mathcal{U}_{i}^{n+1}, 1\right)=0 \text {, and } B\left(\mathcal{U}_{i}^{n+1}, 2\right)<0 \\
& \text { If } \chi_{i}^{n+1}=2 \text {, then } B\left(\mathcal{U}_{i}^{n+1}, 2\right)=0 \text {, and }\left\{\begin{array}{l}
B\left(\mathcal{U}_{i}^{n+1}, 1\right)<0, \\
B\left(\mathcal{U}_{i}^{n+1}, 3\right)>0
\end{array}\right. \\
& \text { If } \chi_{i}^{n+1}=3 \text {, then } B\left(\mathcal{U}_{i}^{n+1}, 3\right)=0, \text { and } B\left(\mathcal{U}_{i}^{n+1}, 2\right)>0
\end{aligned}
$$

For each time step, we apply an adapted Newton method to find an approximate value of $\mathcal{U}=\left(\mathcal{U}_{i}^{n+1}\right)_{i=1, \ldots N_{c v}}$ and $\chi=\left(\chi_{i}^{n+1}\right)_{i=1, \ldots N_{c v}}$, solution of the whole system of equations including the discrete balance equations (18-22) and the equations $B\left(\mathcal{U}_{i}^{n+1}, \chi_{i}^{n+1}\right)=0$. We denote this system of equations by $D(\mathcal{U}, \chi)=0$. Let $\mathcal{U}^{(0)}, \chi^{(0)}$ be an initialization of the unknowns (practically, we use the values obtained from the preceding time step $\mathcal{U}^{(0)}=\left(\mathcal{U}_{i}^{n}\right)_{i=1, \ldots N_{c v}}$ and $\chi^{(0)}=\left(\chi_{i}^{n}\right)_{i=1, \ldots N_{c v}}$.

At iteration $m$ of this adapted Newton's method, let $\mathcal{U}^{(m)}, \chi^{(m)}$ be given. We first compute $\mathcal{U}^{(m+1)}$ from the resolution of the following linear system (for the sake of simplicity, we use in our prototype the method of band matrix Gauss elimination):

$$
\partial_{\mathcal{U}} D\left(\mathcal{U}^{(m)}, \chi^{(m)}\right) \cdot\left(\mathcal{U}^{(m+1)}-\mathcal{U}^{(m)}\right)=-D\left(\mathcal{U}^{(m)}, \chi^{(m)}\right)
$$

in which $\partial_{\mathcal{U}} D(\mathcal{U}, \chi)$ denotes the Jacobian matrix with respect to $x$ of the function $D(\mathcal{U}, \chi)$. Then we define the state $\chi_{i}^{(m+1)}$, for every grid block $i$, by the relations:

$$
\begin{aligned}
& \text { If } \chi_{i}^{(m)}=1 \text { and if } B\left(\mathcal{U}_{i}^{(m+1)}, 2\right)>0 \text {, then } \chi_{i}^{(m+1)}=2 ; \\
& \text { If } \chi_{i}^{(m)}=2 \text { and if } B\left(\mathcal{U}_{i}^{(m+1)}, 1\right)>0 \text {, then } \chi_{i}^{(m+1)}=1 ; \\
& \text { If } \chi_{i}^{(m)}=2 \text { and if } B\left(\mathcal{U}_{i}^{(m+1)}, 3\right)<0 \text {, then } \chi_{i}^{(m+1)}=3 ;
\end{aligned}
$$


If $\chi_{i}^{(m)}=3$ and if $B\left(\mathcal{U}_{i}^{(m+1)}, 2\right)<0$, then $\chi_{i}^{(m+1)}=2$.

Otherwise we set $\chi_{i}^{(m+1)}=\chi_{i}^{(m)}$.

This method, classically used in the oil reservoir simulation setting, appears to be very stable and efficient (the number of iterations needed does not exceed 2 or 3 for most of the time steps), using after convergence a predictor-corrector strategy for the determination of $t^{n+2}-t^{n+1}$. One can reinforce the convergence properties by under-relaxing any iteration step $\mathcal{U}^{(m+1)}-\mathcal{U}^{(m)}$ such that $\left\|\mathcal{U}^{(m+1)}-\mathcal{U}^{(m)}\right\|$ is greater than a desired variation; then the last iterations until convergence do not need to be under-relaxed.

\section{Comparisons between experimental and numerical results}

\subsection{The Mascilia EXPERIMEnT}

We now use the numerical implementation of this model for reproducing experimental results. For that purpose, the "Mascilia" experiment (Castelier, 2001), done at the French establishment for atomic energy (CEA), enables to study the phenomenon of phase transition (water - water vapor) in an initially saturated porous medium: a tank represented by the domain $[-1.5,1.5] \times[-1,1] \times[0,2]$ (lengths in $m$ ) whose walls are isothermal (typically $T_{p}=350 \mathrm{~K}$ ), is filled with sand and initially saturated with water (see figure 2). In the middle of the tank (at the point $x=y=0, z=1$ ) a heating bar (a cylinder with diameter equal to $0.1 \mathrm{~m}$ and height equal to $0.2 \mathrm{~m}$ ), whose power varies from 0 to $1450 \mathrm{~W}$, enables to carry water to boiling. Four hundred thermo- 
Table II. Parameters used for the simulation.

\begin{tabular}{|c|c|c|c|}
\hline & notation & value & units \\
\hline Domain & & {$[0,1.5] \times[0,1] \times[0,2]$} & $m$ \\
\hline Mesh & & $15 \times 12 \times 23$ & - \\
\hline Source term & $\bar{Q}$ & 500 (run1); 900 (run2) & $W$ \\
\hline (punctual source located at $(0,0,1)$ ) & & & - \\
\hline Porosity & $\phi$ & 0.35 & - \\
\hline Permeability & $K$ & $2.5 \times 10^{-12}$ & $m^{2}$ \\
\hline Bulk density of sand & $\rho_{s}$ & 2400 & $k g \cdot m^{-3}$ \\
\hline Heat capacity of sand & $C_{s}$ & 800 & $J \cdot \mathrm{kg}^{-1} \cdot K^{-1}$ \\
\hline Thermal conductivity of wet sand & $\lambda_{\text {wet }}$ & 3 & $W \cdot m^{-1} \cdot K^{-1}$ \\
\hline Thermal conductivity of dry sand & $\lambda_{\mathrm{dry}}$ & 0.3 & $W \cdot m^{-1} \cdot K^{-1}$ \\
\hline
\end{tabular}

couples allow to precisely follow the space and time evolution of the temperature.

In this experiment, three phases are in presence: sand, water and water vapor. In-situ measurements of the properties of the porous medium (porosity, permeability, thermal conductivity, bulk density and heat capacity) have been done by different French laboratories (see table II). Note that measurements of air - water (and not water - water vapor $)$ capillary pressure $\left(P_{c, w-a}\right)$ and relative permeabilities $\left(k_{r l, w-a}\right.$ and $\left.k_{r a, w-a}\right)$ have also been done by another laboratory. However, as we see in section 5, these water - air curves cannot be used without modifications in order to reproduce the Mascilia experiments.

\subsection{RESUlts AND DISCUSSiON}

The data used for the simulation are given in tables II and III. In figure 2, the domain and the boundary conditions are described. The comparisons between experimental and numerical results are essentially 
Table III. Capillary and relative permeabilities curves used for the simulation.

\begin{tabular}{|c|c|c|}
\hline & notation & value \\
\hline Capillary pressure & $P_{c}(S)$ & $\left\{\begin{array}{l}1.70 \times 10^{3}\left(S^{-1.37}-1\right)^{0.37} \text { if } S>0.005 \\
P_{c}(0.005) \text { if } 0<S<0.005\end{array}\right.$ \\
\hline relative permeability of liquid water & $k_{r l}(S)$ & $A_{l} \tanh \left[a_{l}\left(S-b_{l}\right)\right]+B_{l}$ \\
\hline relative permeability of water vapor & $k_{r v}(S)$ & $5 \times A_{v} \tanh \left[a_{v}\left(b_{v}-S\right)\right]+B_{v}$ \\
\hline
\end{tabular}

based on the temperature measurements at various points in the tank.

The temperatures are continuously recorded with respect to the time.

Figures 4 and 5 present the value of the temperature as a function of the $x$ coordinate along the median line at $(y=0, z=1)$ and as a function of the $z$ coordinate $(1 \leq z \leq 2)$ along the vertical line $x=0$ and $y=0$ above the heating bar, at times $t=5000 \mathrm{~s}$ and $t=25000 \mathrm{~s}$. The circles represent experimental curves. These figures show that two areas in the tank can be observed when the imposed power is $\bar{Q}_{0}=500 \mathrm{~W}$ : a two-phase area, quasi isothermal $(375 K)$, included in $[0,0.19] \times[0,0.18] \times[0.85,1.43]$ and an area saturated by the liquid in the rest of the tank. We note that there is a good agreement between the experimental and numerical results: the temperature variation in the tank, the temperature level and the extension of the two-phase area are well evaluated by the simulation whatever the time considered. We observe in figures 6 and 7 which show the liquid saturation field and the temperature field in the plane $y=0$, at time $t=25000 \mathrm{~s}$, that an asymmetry is observed between the values $z \in[1,2]$ and $z \in[0,1]$. The size of the two-phase area is equal to $0.15 \mathrm{~m}$ below the heating source, 
and to $0.43 \mathrm{~m}$ above, as mentioned before.

A similar agreement between experimental and numerical results is again observed as $\bar{Q}_{0}=900 \mathrm{~W}$. Figures 8 and 9 present the value of the temperature as a function of the $x$ coordinate along the median line at $(y=0, z=1)$ and as a function of the $z$ coordinate along the vertical line above the heating bar at time $t=5000 \mathrm{~s}$ and $t=25000 \mathrm{~s}$. The circles represent experimental curves. These figures show that there are three areas in the tank when the imposed power is $900 \mathrm{~W}$ :

1. a small dry vapor zone around the heating bar characterized by a brutal increase in the temperature;

2. a two-phase area, quasi isothermal $(375 K)$ whose size is $0.24 m$ in $x$ direction and $0.56 \mathrm{~m}$ in $z$ direction $(1 \leq z \leq 2)$ at time $t=25000 \mathrm{~s}$;

3. an area saturated by the liquid in the rest of the tank.

On figure 9 , one notes that in $z$ direction, at time $t=5000 \mathrm{~s}$, the existence of a dry vapor area is not reproduced by the computational results and the 'numerical' two-phase area is larger than the experimental one. However, the agreement remains acceptable.

Figures 10 and 11 present liquid and water vapor velocity distributions in the plane $(x, z)$ at $y=0$ and at $t=25000 \mathrm{~s}$. The imposed power is $\bar{Q}=900 \mathrm{~W}$. In these figures, the interface between the twophase zone and the saturated zone is plotted as a solid line. The liquid velocity vectors indicate a liquid flow near the condensation front and in the two-phase region. Far away, the liquid movement is very weak. The vapor velocity vectors show a primarily upward movement upon 
the heating bar because of its much lower density. Due to the stronger upward vapor flow, the capillary forces are predominant in the area below the heating bar. The liquid is drawn up by the capillary forces and evaporates completely as it reaches the bottom edge of the heating bar. Upon the heating bar, at the two-phase interface, liquid is formed as the vapor condenses. Part of this liquid flows downward, driven by gravity and capillarity in an attempt to fill again the drying out part of the porous medium. The rest crosses the sub-cooled zone. The same phenomena are observed by (Stubos et al, 1997). The co-current and counter-current flow in the two-phase region lead to a recirculating flow of water (liquid + water vapor) as displayed in figure 12 .

We show in this section that we are able to accurately reproduce the extension of the two-phase zone and the temperature evolution at different locations of the tank with respect to the space and time variables.

\section{Determination of vapor capillary pressure and relative permeabilities}

In this section, we present a method, using the temperature measurements of the "Mascilia" experiment, to fit the capillary pressure curve between water and water vapor, even though there has been neither measurements of pressures nor saturations. Moreover, we present a parametric study to show the influence of the relative permeabilities curves on the length of the two-phase zone. 
Table IV. Capillary pressure and relative permeabilities curves measured with the couple water and air.

\begin{tabular}{lll}
\hline & notation & value \\
\hline Capillary pressure & $P_{c, w-a}(S)$ & $6.81 \times 10^{3}\left(S^{-1.37}-1\right)^{0.37}$ \\
relative permeability of liquid water & $k_{r l, w-a}(S)$ & $A_{l} \tanh \left[a_{l}\left(S-b_{l}\right)\right]+B_{l}$ \\
relative permeability of air & $k_{r a, w-a}(S)$ & $A_{v} \tanh \left[a_{v}\left(b_{v}-S\right)\right]+B_{v}$ \\
\hline$A_{l}=1 /\left[\tanh \left(a_{l} b_{l}\right)+\tanh \left(a_{l}\left(1-b_{l}\right)\right)\right]$ and $A_{v}=1 /\left[\tanh \left(a_{v} b_{v}\right)+\tanh \left(a_{v}\left(1-b_{v}\right)\right)\right]$ \\
$a_{l}=9.24 \quad b_{l}=0.54 \quad a_{v}=7.77 \quad b_{v}=0.37$
\end{tabular}

In "Mascilia" experiment, as mentioned before, the capillary pressure and relative permeabilities data have been measured with the couple water - air at ambient temperature instead of the couple water - water vapor at $100^{\circ} \mathrm{C}$.

The use of water - air curves instead of water - water vapor curves does not permit to meet the experimental results. Indeed, figure 13 presents the value of the temperature as a function of the $z$ coordinate $(1 \leq z \leq 2)$ at time $t=5000 \mathrm{~s}$ and $t=25000 \mathrm{~s}$, using the water - air function (represented on the figure by squares), whereas the imposed heating power is $500 \mathrm{~W}$. In this figure, we observe that:

1. The computed temperature level reached in the two-phase area $(378 K)$ is higher than the measured one, $375.6 K$, which implies a higher simulated temperature gradient in the two-phase area;

2. The simulated length of this area is under evaluated. It is $0.10 \mathrm{~m}$ shorter than the experimental one. Moreover, the anisotropy of the two-phase area is also under evaluated as it is shown by the dashed line in figure 6. 
To get results closer to the experiments, we have been driven to call the water - air capillary pressure and relative permeability curves into question.

So, we propose a method using the experimental measurements of the temperature to estimate and to fit the water - water vapor capillary pressure. Indeed, the numerical results show that in the two-phase area, the pressure of the liquid phase is approximately constant (figure 14 show that $P_{l} \approx P_{l 0}=1.1 \times 10^{5} \mathrm{~Pa}$ when the liquid saturation $S$ is between 0.3 and 1 in this region). In this two-phase area, the temperature is then an implicit function of the vapor pressure $P_{v}$ thanks to the equation $g_{l}\left(P_{l 0}, T\right)=g_{v}\left(P_{v}, T\right)$. Drawing this function (see figure 15), we can then identify $P_{v} \approx 1.117 \times 10^{5} \mathrm{~Pa}$ as well as the magnitude of $P_{c, \text { mean }}(S) \approx P_{v}-P_{l 0}=1700 P a$ for the recorded equilibrium temperature $T_{\text {mean }} \approx 375.6 \mathrm{~K}$ ( $T_{\text {mean }}$ is an average value of the experimental temperature in the two-phase zone). Thus, we deduce a mean value of the numerical liquid saturation in the two-phase region: $S_{\text {mean }} \approx 0.65$. We then search $P_{c}(0.65) \approx 1700$ and we have obtained the following function

$$
P_{c}(S)=\left\{\begin{array}{rll}
.25 \times P_{c, w-a}(S) & \text { if } \quad S \geq 0.005 \\
.25 \times P_{c, w-a}(0.005) & \text { if } \quad 0<S<0.005
\end{array}\right.
$$

A comparison between the two laws $P_{c, w-a}(S)$ and $P_{c}(S)$ is presented on figure 16. Note that the condition $P_{c}(0)<+\infty$ is necessary to reproduce the appearance of a dry vapor zone around the heating bar. Since the extension of this area is relatively small, it cannot be precisely identified from the temperature measurements, and therefore we did not try to accurately fit the maximum threshold of capillary pressure. 
We have then fitted the vapor relative permeability, in order to meet the actual size of the two-phase zone. To that purpose, we have simulated the cases $k_{r v}(S)=\beta k_{r a, w-a}(S)$, with $\beta=2, \beta=5$ and $\beta=10$. We have thus obtained the results presented in Figures 17 and 18. We have finally kept the following curves:

$$
k_{r v}(S)=5 \times k_{r a, w-a}(S) \text { and } k_{r l}(S)=k_{r l, w-a}(S)
$$

\section{Future research directions}

We present here a physical model for handling the two-phase flows with phase transition in an initially saturated porous medium. This model has been successfully approximated using a finite volume numerical method, which can handle the phenomenon of phase appearance / disappearance, in order to reproduce experimental results. We also propose an original method to approximate the capillary pressure curve from the experimental temperature measurements.

This opens new research directions. The main one is to extend the model to thermo-poro-elastic porous media, in order to handle problems of storage in deep sites. This seems to be possible, following Coussy (Coussy, 2004). The numerical approximation of the resulting model must then account for the coupling between mechanics and unsaturated flows in porous media. An on-going research project concerns the use of a finite element method for the stress and strain computation, coupled with the finite volume method presented here. 
Anyway, some experimental work has to be completed in order to obtain a better knowledge of the capillary pressure and the relative permeability in the case of two-phase water - water vapor flow.

\section{Acknowledgements}

The authors would like to acknowledge Olivier Coussy for determinant discussions.

\section{References}

E. Castelier, O. Gerbaux, D. Jamet, P. Locoge, P. Maugis, Rapport de synthèse Mascilia phase I, CEA (centre de Cadarache), 2001.

O. Coussy, Mechanics of porous continua., Wiley, New York, 1995.

O. Coussy, Poromechanics, John Wiley \& Sons, Chichester, 2004.

O. Coussy, R. Eymard and T. Lassabatère, Constitutive modeling of unsaturated drying deformable materials, J. of Engineering Mechanics. 124(6):658-667, 1998.

J. V. Daurelle, F. Topin and R. Occelli, Modeling of coupled heat and mass transfers with phase change in a porous medium: application to superheated steam drying, Numerical Heat transfer, Part A, 33(1):39-63, 1998.

D. A. De Vries, Thermal properties of soils, In W. R. van Wijk (ed.) Physics of Plant Environment, North-Holland Publishing Co.,Amsterdam, 210-235, 1964.

R. Eymard, T. Gallouët, D. Hilhorst, Y. Naït Slimane, Finite volumes and nonlinear diffusion equations, Mathematical Modelling and Numerical Analysis, 32(6):747$761,1998$.

R. Eymard, T. Gallouët, R. Herbin, The finite volume method, Handbook of Numerical Analysis, Ph. Ciarlet J.L Lions eds, Elsevier, 7:715-1022, 2000. 
R. Eymard, R. Herbin, A. Michel, Mathematical study of a petroleum-engineering scheme, Mathematical Modelling and numerical Analysis, 37(6):937-972, 2003.

R. Ghafir, Modélisation et simulation numérique de l'ébullition par convection forcée dans un milieux poreux, Thesis of the University of Marne-la-Vallée (in French), France, 2000.

J. E. Kelly, M. L. Schwarz, J. T. Hitchcock, Heat-transfer characteristics of dry porous particulate beds with internal heat generation, In proc of ASME-JSME thermal Engng Joint conf, 4:83-91, 1983.

S. Medhekar, M. Abolfadl and T.G. Theofanous, Triggering and propagation of steam explosions, Nucl. Eng. Des, 126(1):23-39, 1991.

M. Najjari, S. B. Nasrallah Numerical study of boiling with mixed convection in a vertical porous layer, Int J. of Thermal Sciences, 41:913-925, 2002.

P. S. Ramesh and K.E. Torrance, Boiling in porous layer heated from bellow: effects of natural convection and a moving liquid/two phase interface, J. Fluid Mech, 257:289-309, 1993.

W. M. Rohsenow, J. P. Harnett, Y. I. Cho, Handbook of heat transfer, McGraw-Hill, Third edition, 1998.

B. A. Schrefler and Zhan Xiaoyong A fully coupled model for water flow and air flow in deformable porous media, Water Resour. Res, 29:155-167, 1993.

A. K. Stubos, C. Pérez Caseiras, J. M. Buchelin and N. K. Kanellopoulos Numerical investigation of vapor liquid flow and heat transfer in capillary porous media, Numerical Heat Transfer. Part A 31:143-166, 1997.

P. Toulhoat, P. Chaix, Prédire le comportement à long terme des colis de déchets, Clefs du CEA, 46, Printemps 2002.

C. Y. Wang and C. Beckermann, A two-phase mixture model of liquid-gas flow and heat transfer in capillary porous media-I. Formulation, Int J. Heat and Mass Transfer, 36(11):2747-2758, 1993.

S. Whitaker, Coupled transport in multiphase systems: a theory of drying, Advances in Heat Transfers, Academic press New York, 31:1-102, 1998.

A.W. Woods, Liquid and vapor flow in superheated rock, Ann Rev. Fluids Mech. 31:171-199, 1999. 


\section{Appendix}

\section{A. Comparisons between analytical and numerical results}

Mathematical results on finite volume schemes have shown the good numerical properties of the numerical scheme presented in Section 3. In particular, the convergence of this method applied to two phase flow in porous media has been extensively studied (see for example (Eymard et al, 2000; Eymard et al, 2003)), and its convergence to the solution of the Stefan problem has also been proven (Eymard et al, 1998). Note that these properties are relevant in the case of Model ((11), (12), (13), (14)), which includes both physical features. We propose in this appendix a comparison of numerical results and analytical ones for this model, under the assumptions given in Table V. Equation (11) gives in this case

$$
\nabla \cdot\left(-\nabla P_{l}+\rho_{f} \mathbf{g}\right)=0
$$

Thus, Equation (27) is now decoupled from the other equations, and we then set $\mathbf{v}_{\mathbf{f}}=\frac{K}{\mu_{f}}\left(-\nabla P_{l}+\rho_{f} \mathbf{g}\right)$. Since we assume that $P_{c}(S)=0$ holds, there is no need to further consider Equation (13). We now consider that the problem holds in the horizontal 1D domain $[0, D]$, and that a constant pressure $P_{0}$ is imposed at $x=0$, and a constant pressure $P_{1}$ is imposed at $x=D$; in this case, $\mathbf{v}_{\mathbf{f}}$ reduces to the scalar constant $v_{f}=\frac{K}{\mu_{f}} \frac{P_{0}-P_{1}}{D}$, and the fluid pressure is given by $P_{l}(x, t)=P_{0}+\frac{P_{1}-P_{0}}{D} x$. Consequently, due to the linear interpolation of the pressure, the numerical scheme (presented in Section 3) exactly 
Table V. Physical assumptions for the analytical solution

\begin{tabular}{lll}
\hline & notation & assumptions \\
\hline Liquid heat capacity & $C_{l 0}$ & constant $C_{f}$ \\
Liquid bulk density & $\rho_{l}\left(P_{l}, T\right)$ & constant $\rho_{f}$ \\
Liquid viscosity & $\mu_{l}(T)$ & constant $\mu_{f}$ \\
Liquid enthalpy & $h_{l}\left(P_{l}, T\right)$ & $C_{f}\left(T-T_{0}\right)$ \\
Liquid entropy & $\eta_{l}\left(P_{l}, T\right)$ & $C_{f} \log \left(T / T_{0}\right)$ \\
Liquid Gibbs potential & $g_{l}\left(P_{l}, T\right)$ & $h_{l}\left(P_{l}, T\right)-T \eta_{l}\left(P_{l}, T\right)$ \\
Vapor heat capacity & $C_{v 0}$ & constant $C_{f}$ \\
Vapor bulk density & $\rho_{v}\left(P_{v}, T\right)$ & constant $\rho_{f}$ \\
Vapor viscosity & $\mu_{v}$ & constant $\mu_{f}$ \\
Latent heat at $T_{0}$ & $L_{0}$ & constant \\
Vapor enthalpy & $h_{v}\left(P_{v}, T\right)$ & $L_{0}+C_{f}\left(T-T_{0}\right)$ \\
Vapor entropy & $\eta_{v}\left(P_{v}, T\right)$ & $L_{0} / T_{0}+C_{f} \log \left(T / T_{0}\right)$ \\
Vapor Gibbs potential & $g_{v}\left(P_{v}, T\right)$ & $h_{v}\left(P_{v}, T\right)-T \eta_{v}\left(P_{v}, T\right)$ \\
Porosity & $\phi$ & constant \\
Permeability & $K$ & constant \\
Heat capacity of sand & $C_{s}$ & constant 0 \\
Thermal conductivity of wet sand & $\lambda_{\text {wet }}$ & constant $\lambda$ \\
Thermal conductivity of dry sand & $\lambda_{\mathrm{dry}}$ & constant $\lambda$ \\
Heat source term & $\bar{Q}$ & constant 0 \\
Capillary pressure & $P_{c}(S)$ & constant 0 \\
Relative permeability of liquid water & $k_{r l}(S)$ & $S$ \\
Relative permeability of water vapor & $k_{r v}(S)$ & $1-S$ \\
\hline
\end{tabular}

reproduces the analytical solution for the common pressure of both fluids and for the fluid velocity.

Thanks to the assumptions, we now write the problem in an adimensional form. To that purpose, we define $v=\frac{\lambda}{\phi \rho_{f} C_{f} D}$, the Péclet number $\mathcal{P}=\frac{v_{f}}{\phi v}$, and we set $\bar{x}=\frac{x}{D}, \bar{t}=\frac{v t}{D}, \bar{L}=\frac{L_{0}}{C_{f} T_{0}}, \bar{T}(\bar{x}, \bar{t})=\frac{1}{T_{0}} T\left(D \bar{x}, \frac{D \bar{t}}{v}\right)$, $\bar{S}(\bar{x}, \bar{t})=S\left(D \bar{x}, \frac{D \bar{t}}{v}\right)$, and $\bar{H}(\bar{T}, \bar{S})=\bar{T}+\bar{L}(1-\bar{S})$. Equation (12) leads to

$$
\frac{\partial \bar{H}}{\partial \bar{t}}+\mathcal{P} \frac{\partial \bar{H}}{\partial \bar{x}}-\frac{\partial^{2} \bar{T}}{\partial \bar{x}^{2}}=0
$$


Moreover, Equations and Inequations (14) give

state 1 : no vapor phase, $\bar{S}=1$ and $\bar{T}<1$

state 2 : liquid-vapor equilibrium, $\bar{T}=1$ and $0<\bar{S}<1$

state 3 : no liquid phase, $\bar{S}=0$ and $\bar{T}>1$.

System (28)-(29) is in fact, in this particular case, the so-called "enthalpy formulation" of the Stefan problem, which is also used in (Wang et al, 1993) in the general case. Indeed, thanks to (29), it is possible to express the temperature $\bar{T}$ as a function with respect to $\bar{H}$. For that purpose, we set $\varphi(\bar{H})=\bar{H}$ for $\bar{H} \leq 1$ (it corresponds to $\bar{S}=1$ and $\bar{T}<1), \varphi(\bar{H})=1$ for $1 \leq \bar{H} \leq 1+\bar{L}$ (it corresponds to $0<\bar{S}<1$ and $\bar{T}=1$ ), and $\varphi(\bar{H})=\bar{H}-\bar{L}$ for $1+\bar{L} \leq \bar{H}$ (it corresponds to $\bar{S}=0$ and $\bar{T}>1$ ). The equation to be solved is therefore

$$
\frac{\partial \bar{H}}{\partial \bar{t}}+\mathcal{P} \frac{\partial \bar{H}}{\partial \bar{x}}-\frac{\partial^{2} \varphi(\bar{H})}{\partial \bar{x}^{2}}=0
$$

The saturation is then obtained from $\bar{H}$ by the relation $\bar{S}=\psi(\bar{H})=$ $1-(\bar{H}-\varphi(\bar{H})) / \bar{L}$.

Then a function $\bar{H}(\bar{x}, \bar{t})$ is a weak solution of the equation (30) if this equation is strongly verified in the space-time domain where $\bar{H}$ is a regular function, if the temperature $\bar{T}=\varphi(\bar{H})$ is a continuous function of the space and time variables, and if the Rankine Hugoniot jump relation, expressed by $\dot{X}(\bar{t})\left(\bar{H}(X(\bar{t}), \bar{t})^{-}-\bar{H}(X(\bar{t}), \bar{t})^{+}\right)=$ $\mathcal{P}\left(\bar{H}(X(\bar{t}), \bar{t})^{-}-\bar{H}(X(\bar{t}), \bar{t})^{+}\right)-\left(\frac{\partial \varphi(\bar{H})}{\partial \bar{x}}(X(\bar{t}), \bar{t})^{-}-\frac{\partial \varphi(\bar{H})}{\partial \bar{x}}(X(\bar{t}), \bar{t})^{+}\right)$, is satisfied along the discontinuities lines $\bar{x}=X(\bar{t})$ of $\bar{H}$. We set $\bar{v}=\mathcal{P}+1$, and we consider for any value $A$ such that $0 \leq A \leq 1$, the function $\bar{H}$ 
given by

$$
\begin{aligned}
\bar{H}(\bar{x}, \bar{t}) & =(1+\bar{L}-A) \exp (\bar{v} \bar{t}-\bar{x})+A, \text { for } 0 \leq \bar{x}<\bar{v} \bar{t} \\
\bar{H}(\bar{x}, \bar{t}) & =(1-A) \exp (\bar{v} \bar{t}-\bar{x})+A, \text { for } \bar{v} \bar{t}<\bar{x} .
\end{aligned}
$$

We have to verify that the function $\bar{H}$ given by (31) is indeed a weak solution to (30). In both domains $0 \leq \bar{x}<\bar{v} \bar{t}$ and $\bar{v} \bar{t}<\bar{x}$ where the function $\bar{H}$ is regular, the left hand side of (30) is proportional to $\bar{v}-\mathcal{P}-1=0$, thanks to the definition of $\bar{v}$. This proves that the equation is strongly satisfied in these domains. The temperature field defined by $\bar{T}(\bar{x}, \bar{t})=\varphi(\bar{H}(\bar{x}, \bar{t}))$ is continuous with respect to the time and space variables, since the only discontinuity on $\bar{H}(\bar{x}, \bar{t})$ occurs with $\bar{T}=1$. The Rankine-Hugoniot relation at the discontinuity line $X(\bar{t})=\bar{v} \bar{t}$ is expressed in this case by $\bar{v} \bar{L}=\mathcal{P} \bar{L}+(1+\bar{L}-A)-(1-A)$, which holds. Therefore the criteria for a weak solution are all satisfied by the function $\bar{H}$ given by (31). We can then express the saturation and the temperature in both domains, by the relations $\bar{S}(\bar{x}, \bar{t})=0$ and $\bar{T}(\bar{x}, \bar{t})=(1+\bar{L}-A) \exp (\bar{v} \bar{t}-\bar{x})-\bar{L}+A$ for all $\bar{x}$ such that $0 \leq \bar{x}<\bar{v} \bar{t}$, $\bar{S}(\bar{x}, \bar{t})=1$ and $\bar{T}(\bar{x}, \bar{t})=(1-A) \exp (\bar{v} \bar{t}-\bar{x})+A$ for all $\bar{x}$ such that $\bar{v} \bar{t}<\bar{x}$. Note that the function $\bar{S}(\bar{x}, \bar{t})$ is discontinuous for $\bar{v} \bar{t}=\bar{x}$, which is possible because we have assumed that there were no capillary pressure.

To compare analytical and computational results, we will consider two cases: $A=0$ and $A=1$. Let us first consider the case $A=0$ and the data given in table VI. The analytical solution is for $0<\bar{x}<\bar{v} \bar{t}$, $\bar{T}(\bar{x}, \bar{t})=2 \exp (\bar{v} \bar{t}-\bar{x})-1$ and $\bar{S}(\bar{x}, \bar{t})=0$ and for $\bar{x}>\bar{v} \bar{t}, \bar{T}(\bar{x}, \bar{t})=$ $\exp (\bar{v} \bar{t}-\bar{x})$ and $\bar{S}(\bar{x}, \bar{t})=1$. In this case, the length of the domain 
Table VI. Data for the comparison between analytical and computational solution

\begin{tabular}{ccccccccc}
\hline $\mathrm{A}$ & $\bar{L}$ & $\bar{v}$ & $\bar{S}(\bar{x}, 0)$ & $\bar{S}(0, \bar{t})$ & $\bar{S}(1, \bar{t})$ & $\bar{T}(\bar{x}, 0)$ & $\bar{T}(0, \bar{t})$ & $\bar{T}(1, \bar{t})$ \\
\hline 0 & 1 & 1.5 & 1 & 0 & 1 & $\exp (-\overline{\mathrm{x}})$ & $2 \exp (\overline{\mathrm{v}} \overline{\mathrm{t}})-1$ & $\exp (\overline{\mathrm{v}} \overline{\mathrm{t}}-1)$ \\
1 & 1 & 1.5 & 1 & 0 & 1 & 1 & $\exp (\overline{\mathrm{v}} \overline{\mathrm{t}})$ & 1 \\
\hline
\end{tabular}

such that $\bar{T}(\bar{x}, \bar{t})=1$ is reduced to 0 (this is a classical property of the Stefan Problem). Using the numerical scheme presented in Section 3, we obtain the curves given in Figures 19 and 20 (in adimensional time, space and variables). We first remark that the curves obtained for 100 grid blocks cannot be distinguished from the analytical solution. Secondly, we notice that, even in the case of a coarse discretization with 20 grid blocks, the calculation gives acceptable results.

Let us now consider the case $A=1$. The data for this case are given in table VI. The analytical solution is for $0<\bar{x}<\bar{v} \bar{t}, \bar{T}(\bar{x}, \bar{t})=$ $\exp (\bar{v} \bar{t}-\bar{x})$ and $\bar{S}(\bar{x}, \bar{t})=0$ and for $\bar{x}>\bar{v} \bar{t}, \bar{T}(\bar{x}, \bar{t})=1$ and $\bar{S}(\bar{x}, \bar{t})=1$. In this case, the domain such that $\bar{T}(\bar{x}, 0)=1$ is the whole domain. Classically, this domain progressively vanishes as times goes on. We find again in Figures 21 and 22 that the comparison in this case between the numerical (100 grid blocks and 20 grid blocks) and the analytical results is very good. 


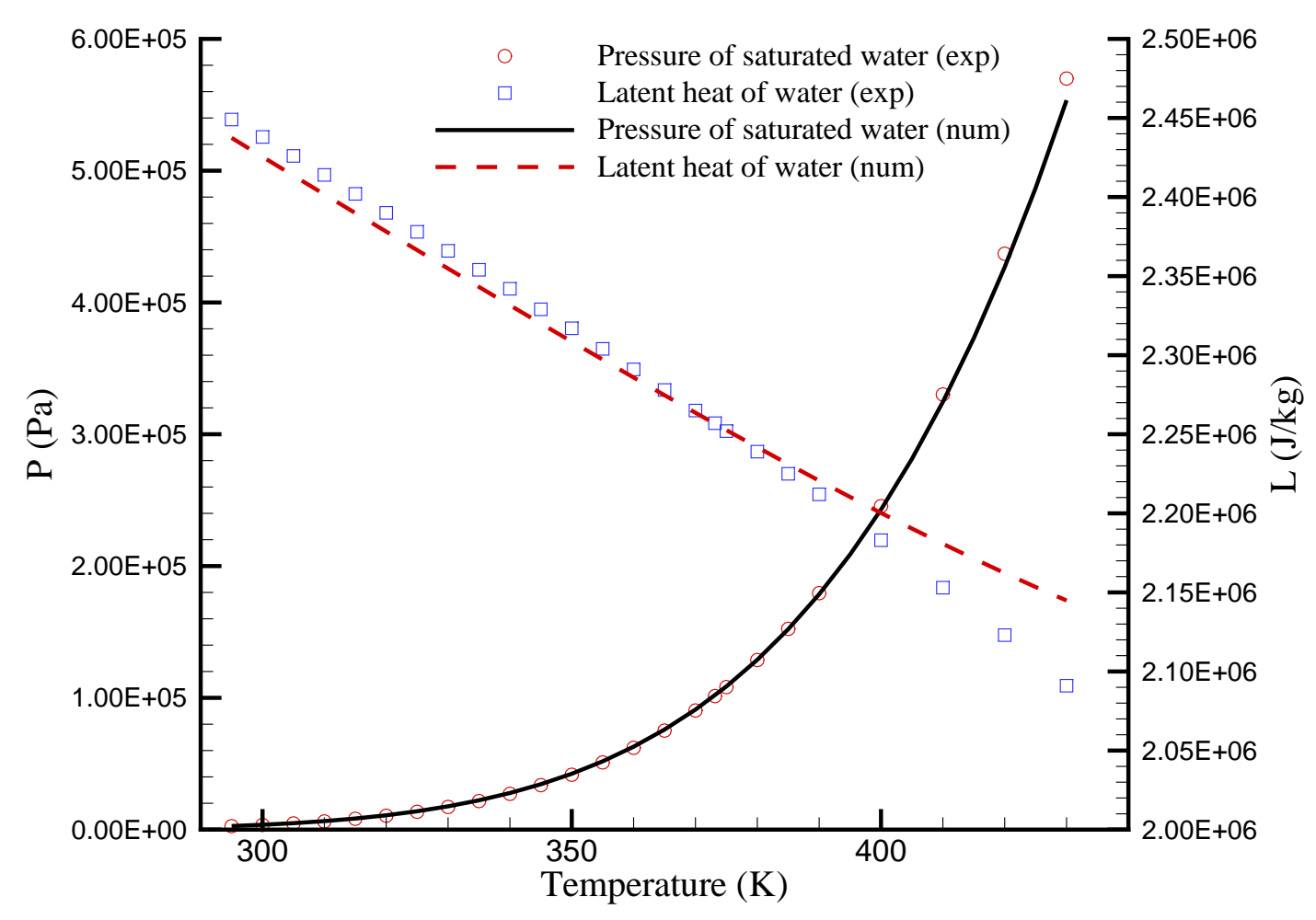

Figure 1. Pressure of saturated water and latent heat of water as a function of the temperature. The circles and the squares result from the experimental data (Rohsenow et al, 1998). The solid and the dashed lines results from the computational $g_{v}(P(T), T)=g_{l}(P(T), T)$. 


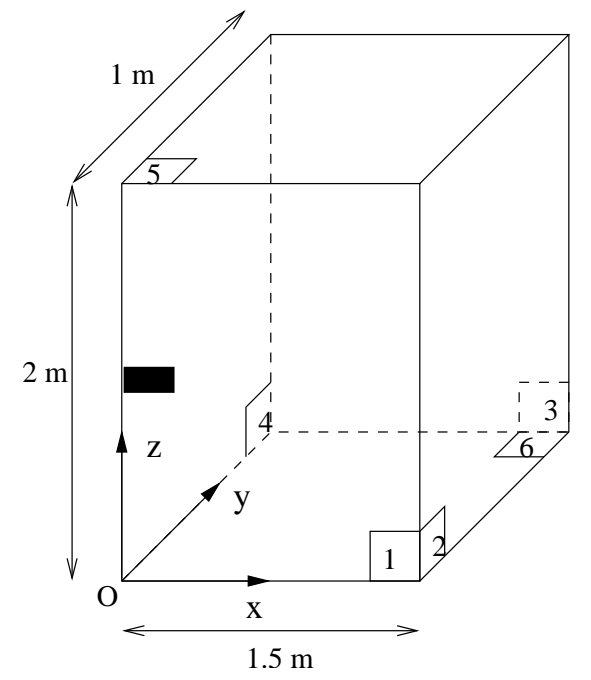

Heating bar

$1 \quad 4 \quad$ Symmetry conditions

2 Permeable wall at uniform temperature: $77^{\circ} \mathrm{C}$

3 Impermeable wall at uniform temperature: $79^{\circ} \mathrm{C}$

65 Impermeable wall at uniform temperature: $77^{\circ} \mathrm{C}$

Figure 2. Field of study and boundary condition description. Since the device is symmetric with respect to the planes of equations $x=0$ and $y=0$ we can only consider a quarter of the domain, introducing zero mass and energy fluxes conditions at the symmetry planes. Note that wall 2 is permeable: the imposed liquid saturation is 1 and the imposed pressure is hydrostatic. The initial conditions are: $S=1$, $T=350 K$ under a hydrostatic pressure. 

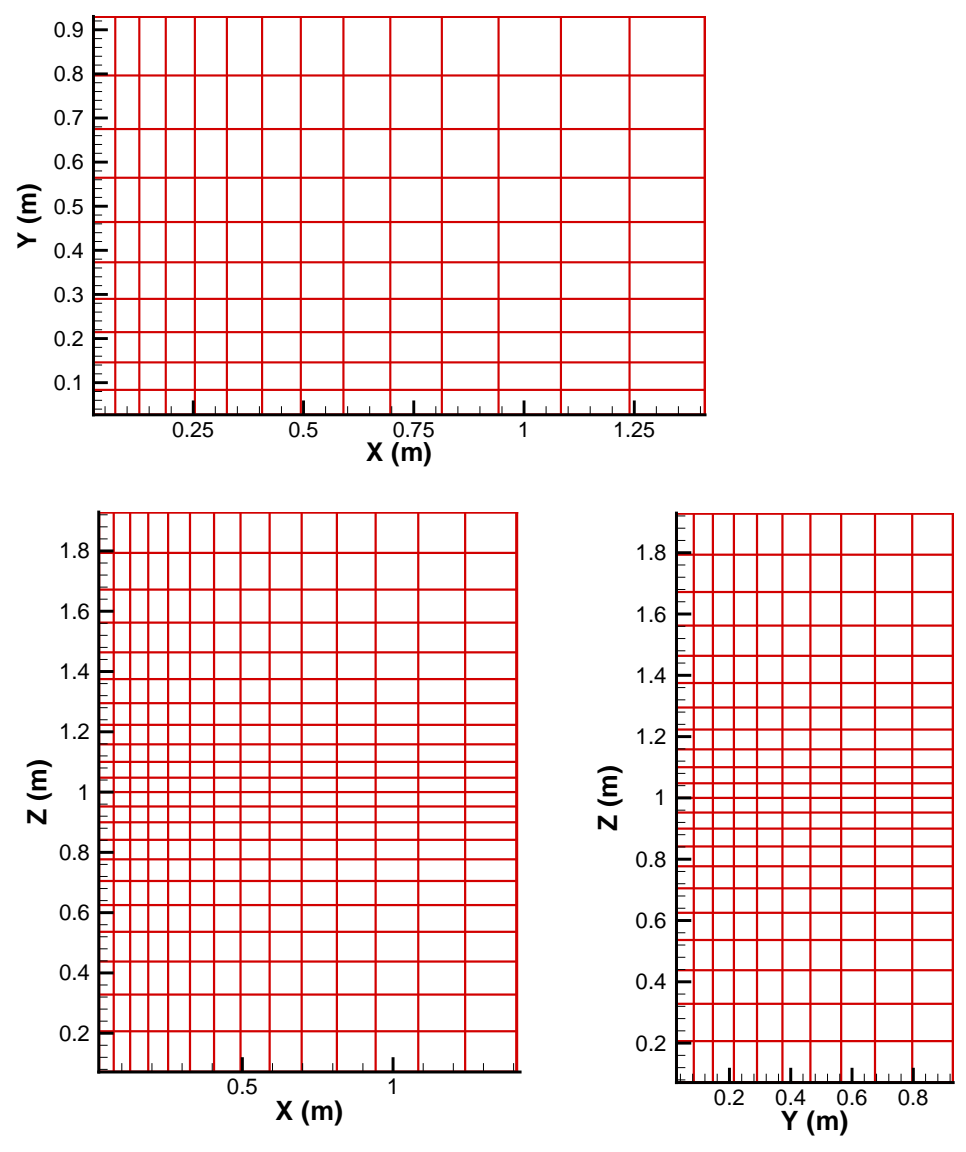

Figure 3. Mesh description. 


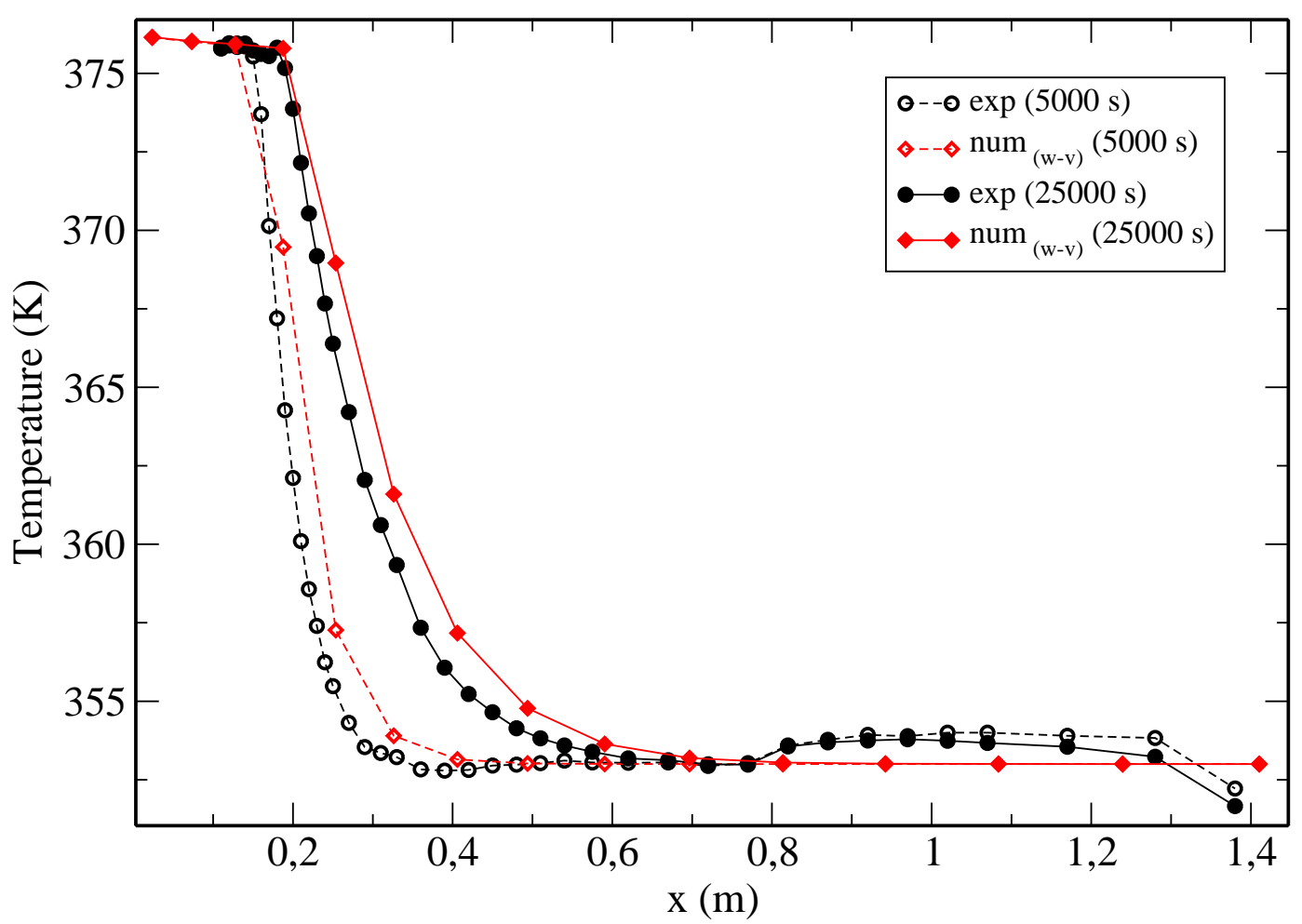

Figure 4. Temperature profiles along axis $x$ at $(y, z)=(0,1)$ and at times $t=5000 \mathrm{~s}$ and $t=25000 \mathrm{~s}$. Imposed power $\bar{Q}_{0}=500 \mathrm{~W}$. 


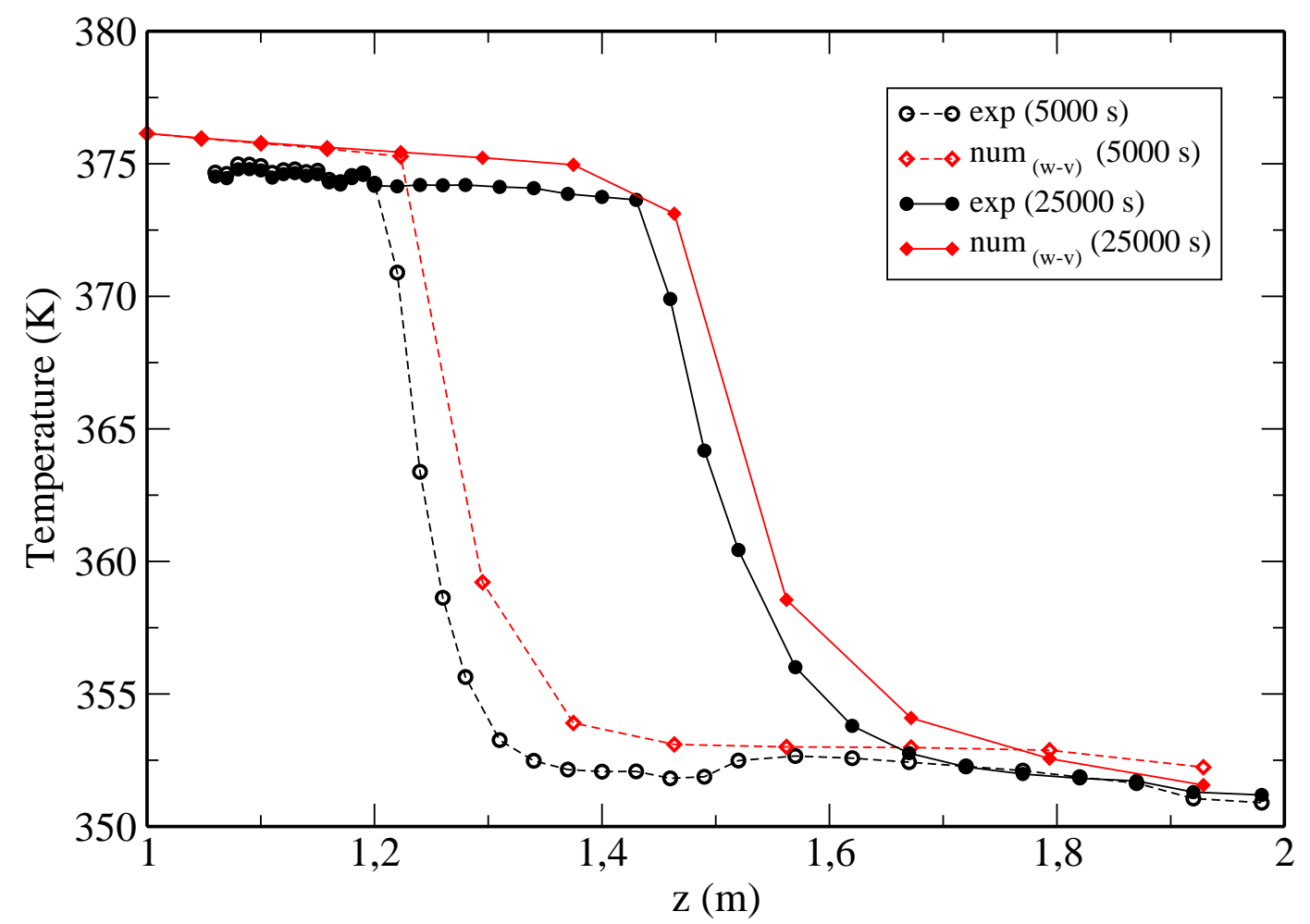

Figure 5. Temperature profiles along axis $z(1 m \leq z \leq 2 m)$ at $(x, y)=(0,0)$ and at times $t=5000 \mathrm{~s}$ and $t=25000 \mathrm{~s}$. Imposed power $\bar{Q}_{0}=500 \mathrm{~W}$. 


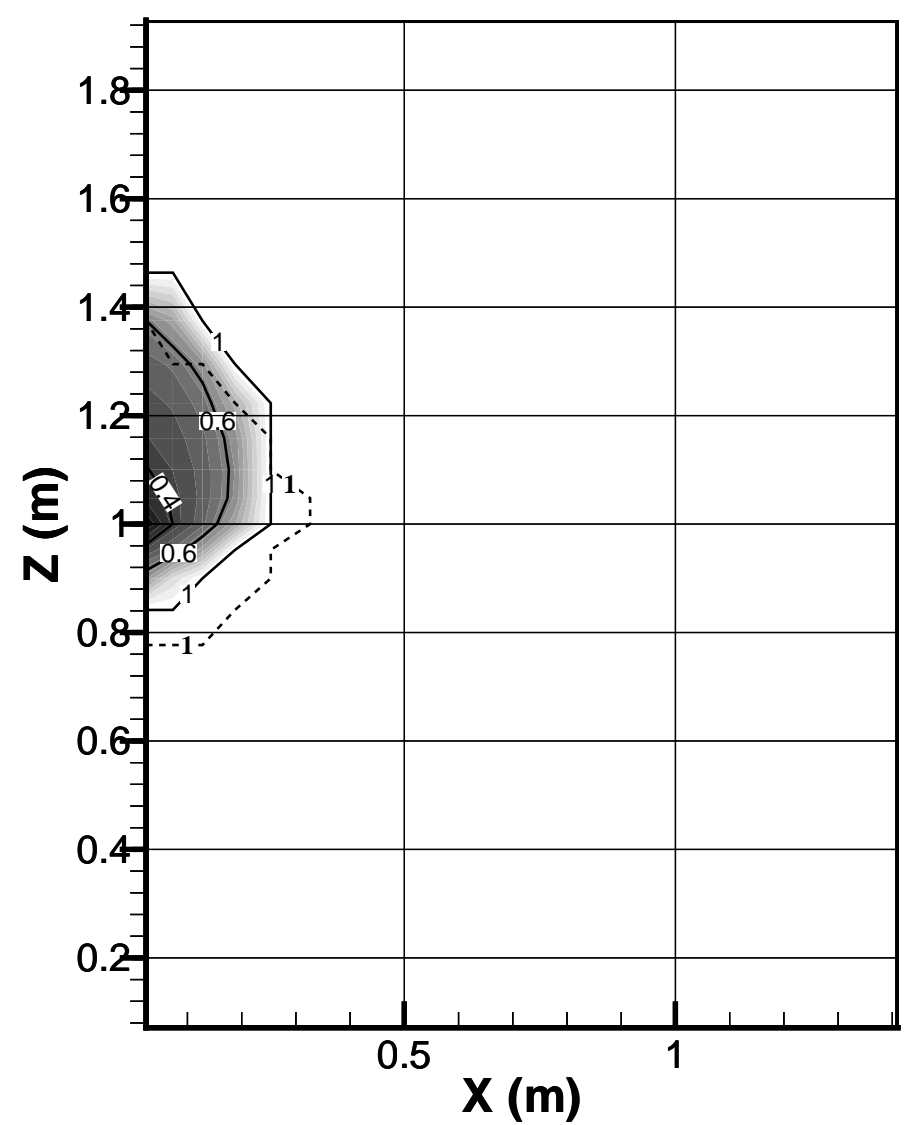

Figure 6. Liquid saturation field in the plane $(x, z)$ at $y=0$, at time $t=25000 \mathrm{~s}$. Imposed power $\bar{Q}_{0}=500 \mathrm{~W}$. The three solid lines represent particular isovalues of liquid saturation $(S=0.4,0.6,1)$. The dashed line represents the saturation isovalue $S=1$ computed in the same conditions as above, but with the experimental capillary pressure $P_{c, w-a}$ and the experimental vapor permeability $k_{r v, w-a}$. 


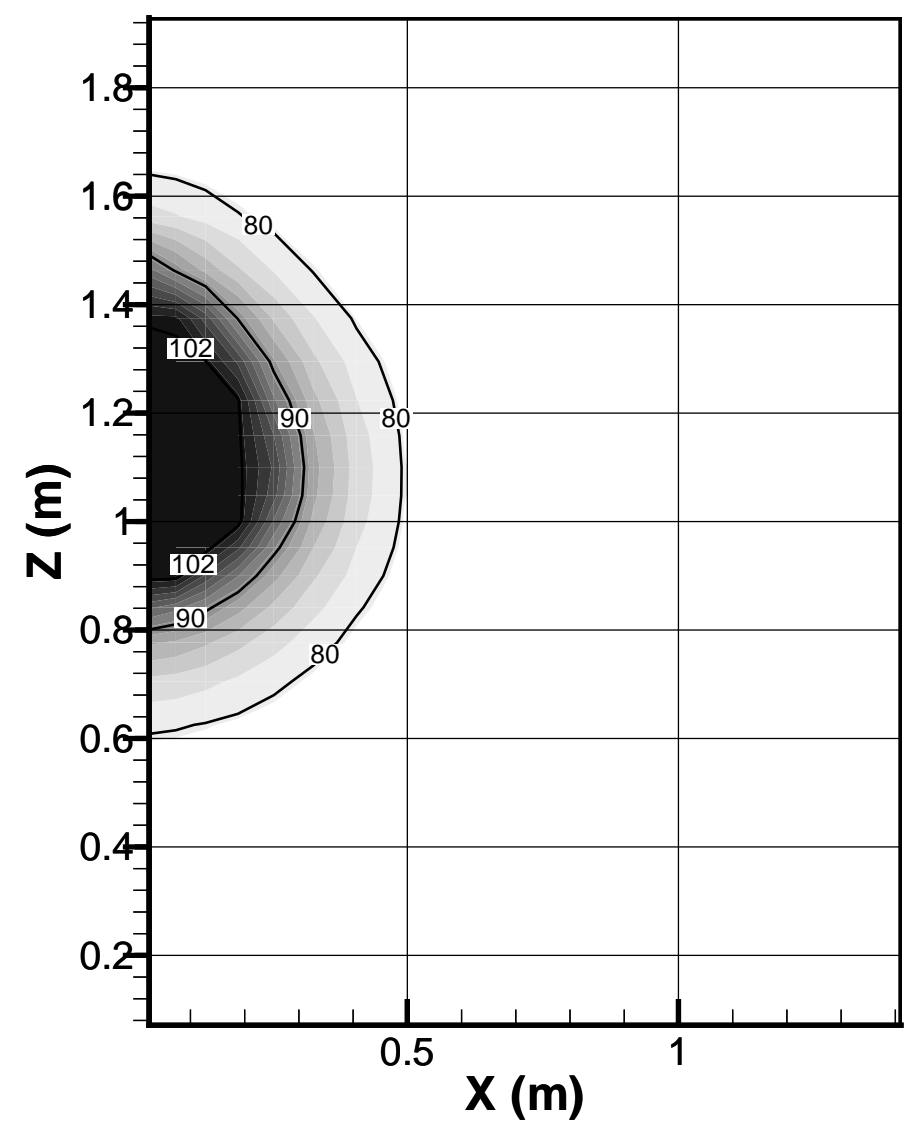

Figure \%. Temperature field in the plane $(x, z)$ at $y=0$ at time $t=25000 \mathrm{~s}$. Imposed power $\bar{Q}_{0}=500 \mathrm{~W}$. The solid lines represent isotherms in degrees Celsius. 


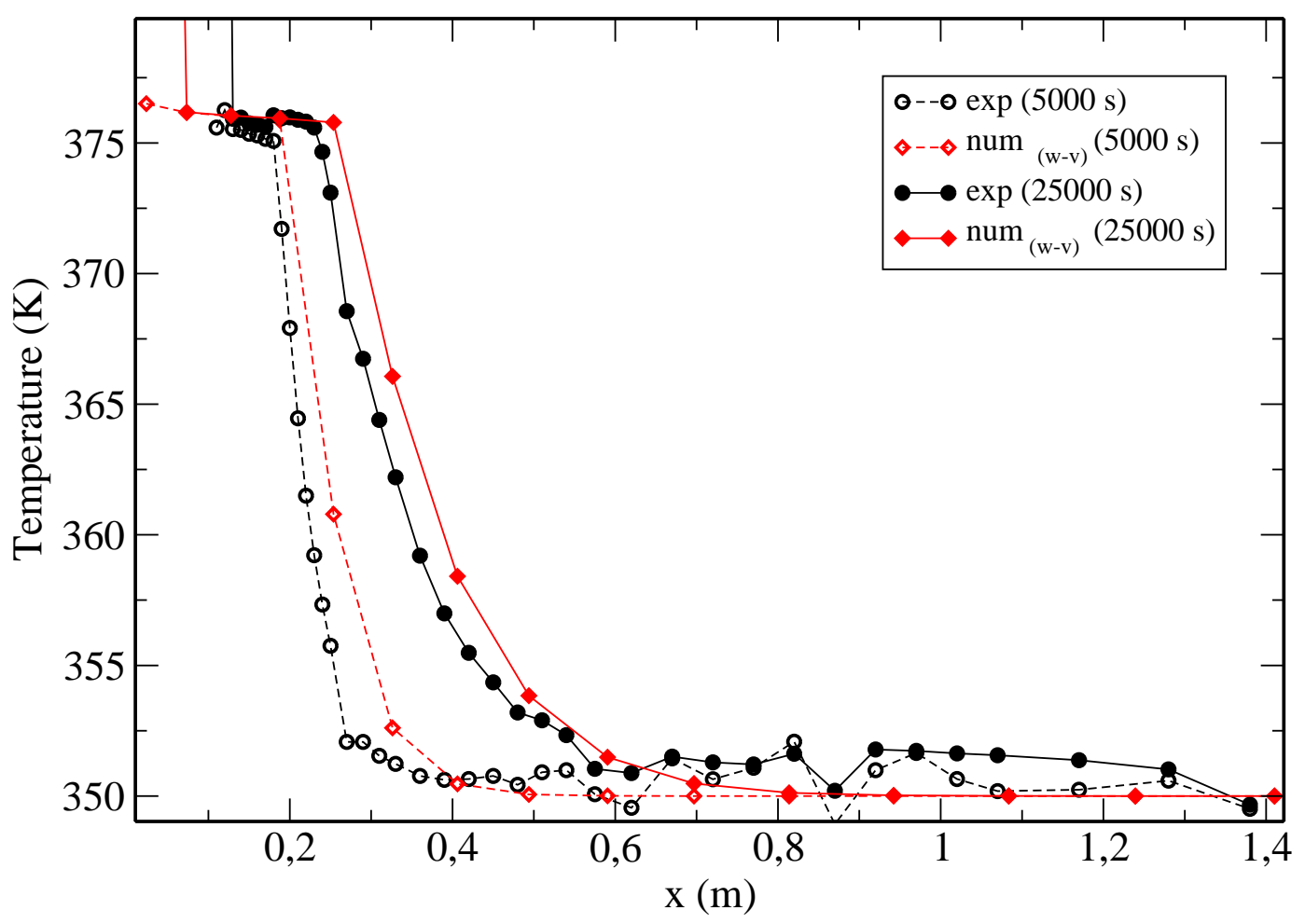

Figure 8. Temperature profiles along axis $x$ at $(y, z)=(0,1)$ and at times $t=5000 \mathrm{~s}$ and $t=25000 \mathrm{~s}$. Imposed power $\bar{Q}_{0}=900 \mathrm{~W}$. 


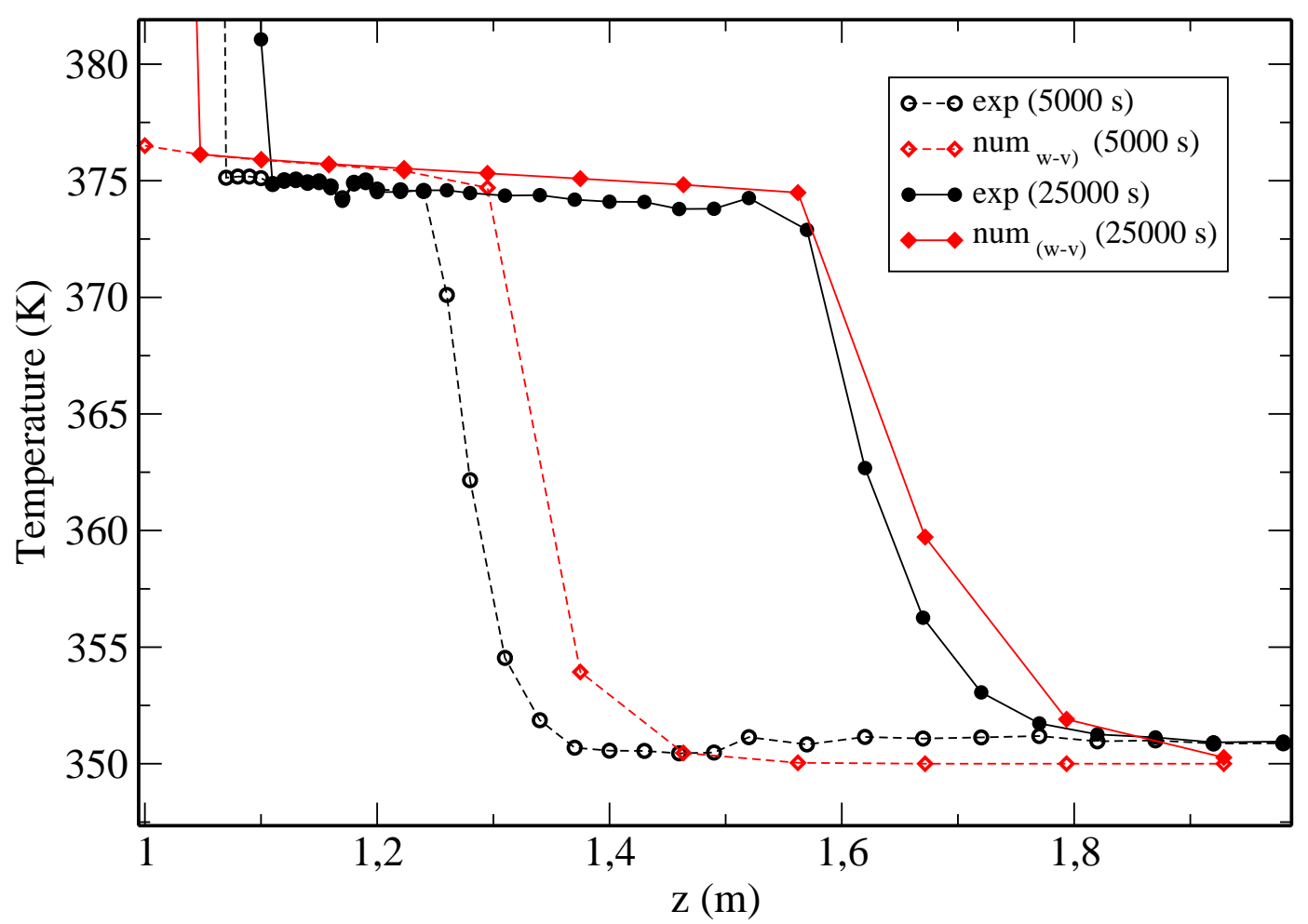

Figure 9. Temperature profiles along axis $z(1 m \leq z \leq 2 m)$ at $(x, y)=(0,0)$ and at times $t=5000 \mathrm{~s}$ and $t=25000 \mathrm{~s}$. Imposed power $\bar{Q}_{0}=900 \mathrm{~W}$. 


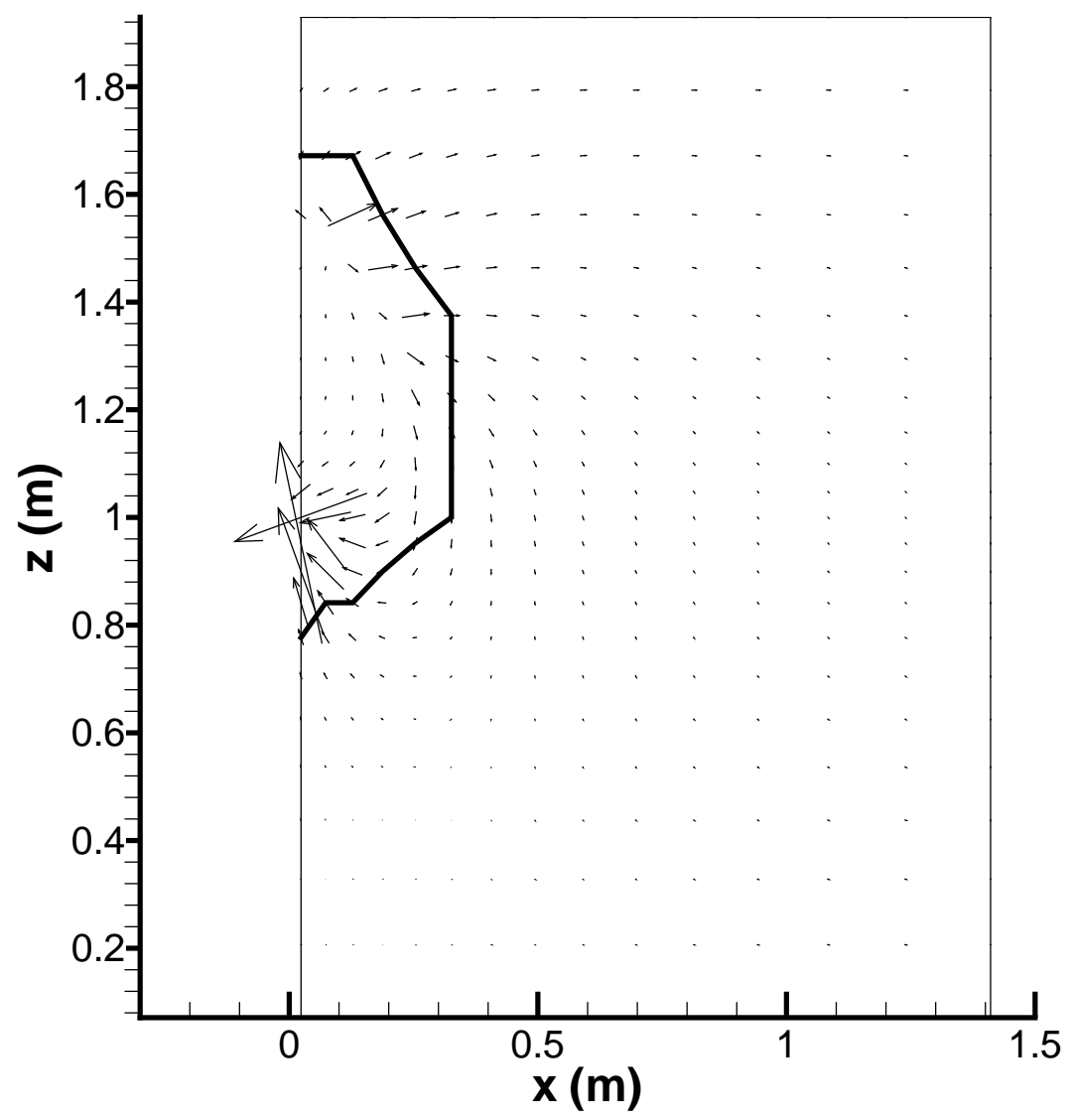

Figure 10. Distribution of liquid velocities in the plane $(x, z)$, at $y=0$, at time $t=25000 \mathrm{~s}$. The velocity vector are represented by arrows centered on the computational points. Imposed power $\bar{Q}_{0}=900 \mathrm{~W}$. The solid line represents isovalue $S=1$ of liquid saturation. 


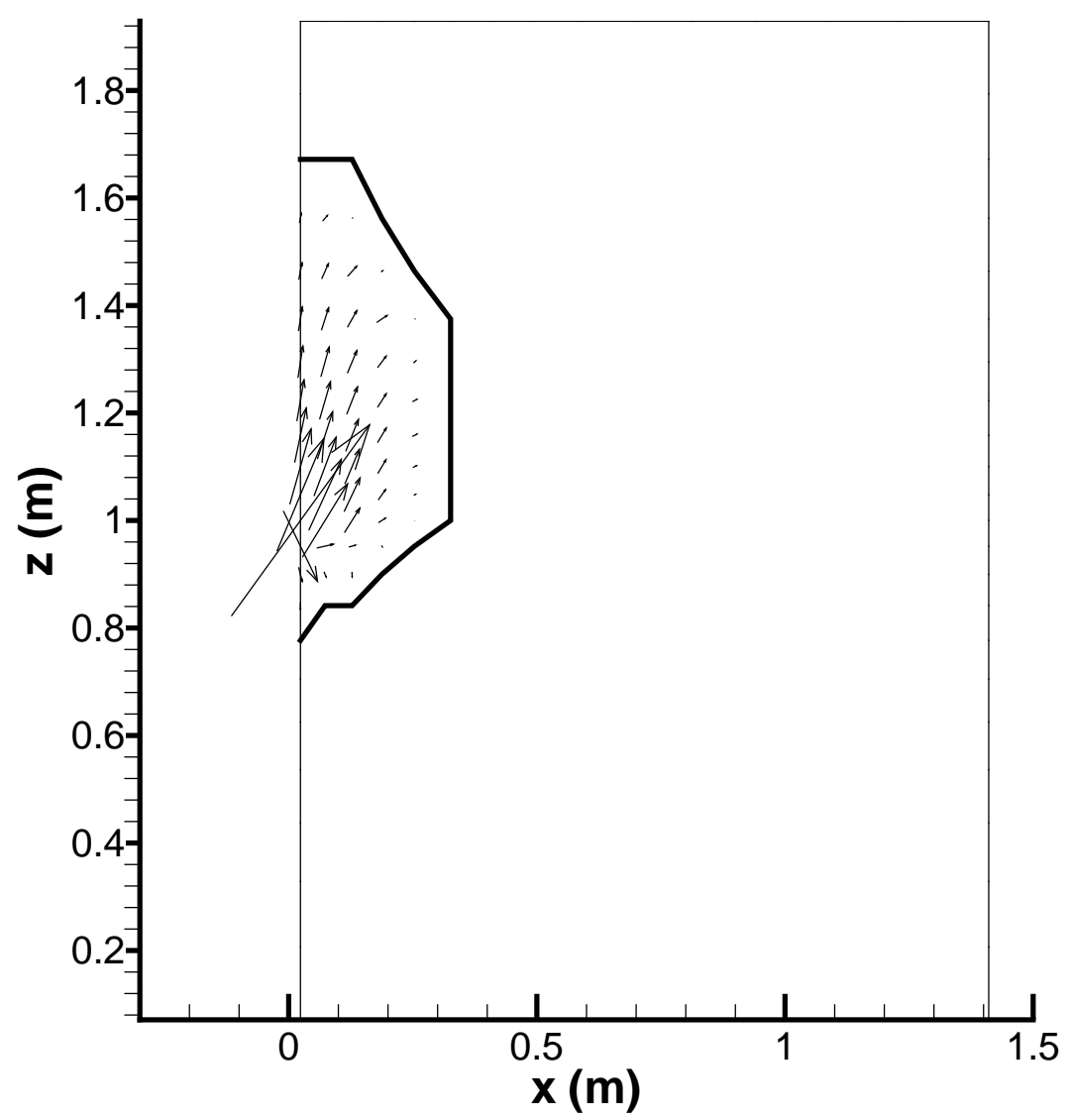

Figure 11. Distribution of water vapor velocities in the plane $(x, z)$, at $y=0$, at time $t=25000 \mathrm{~s}$. The velocity vector are represented by arrows centered on the computational points. Imposed power $\bar{Q}_{0}=900 \mathrm{~W}$. The solid line represents isovalue $S=1$ of liquid saturation. 


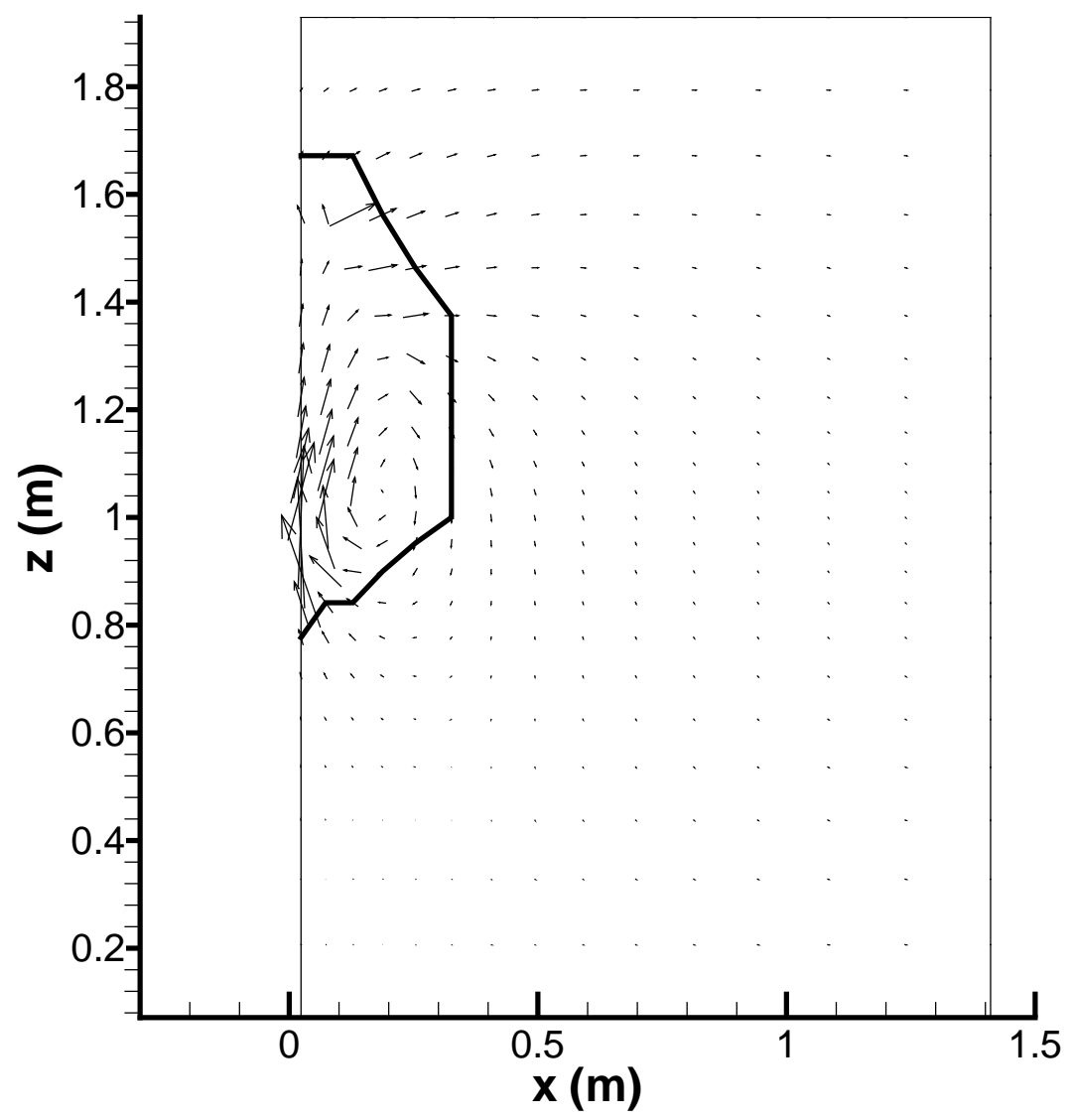

Figure 12. Distribution of water velocities in the plane $(x, z)$, at $y=0$, at time $t=25000 \mathrm{~s}$. The velocity vector are represented by arrows centered on the computational points. Imposed power $\bar{Q}_{0}=900 \mathrm{~W}$. The solid line represents isovalue $S=1$ of liquid saturation. 


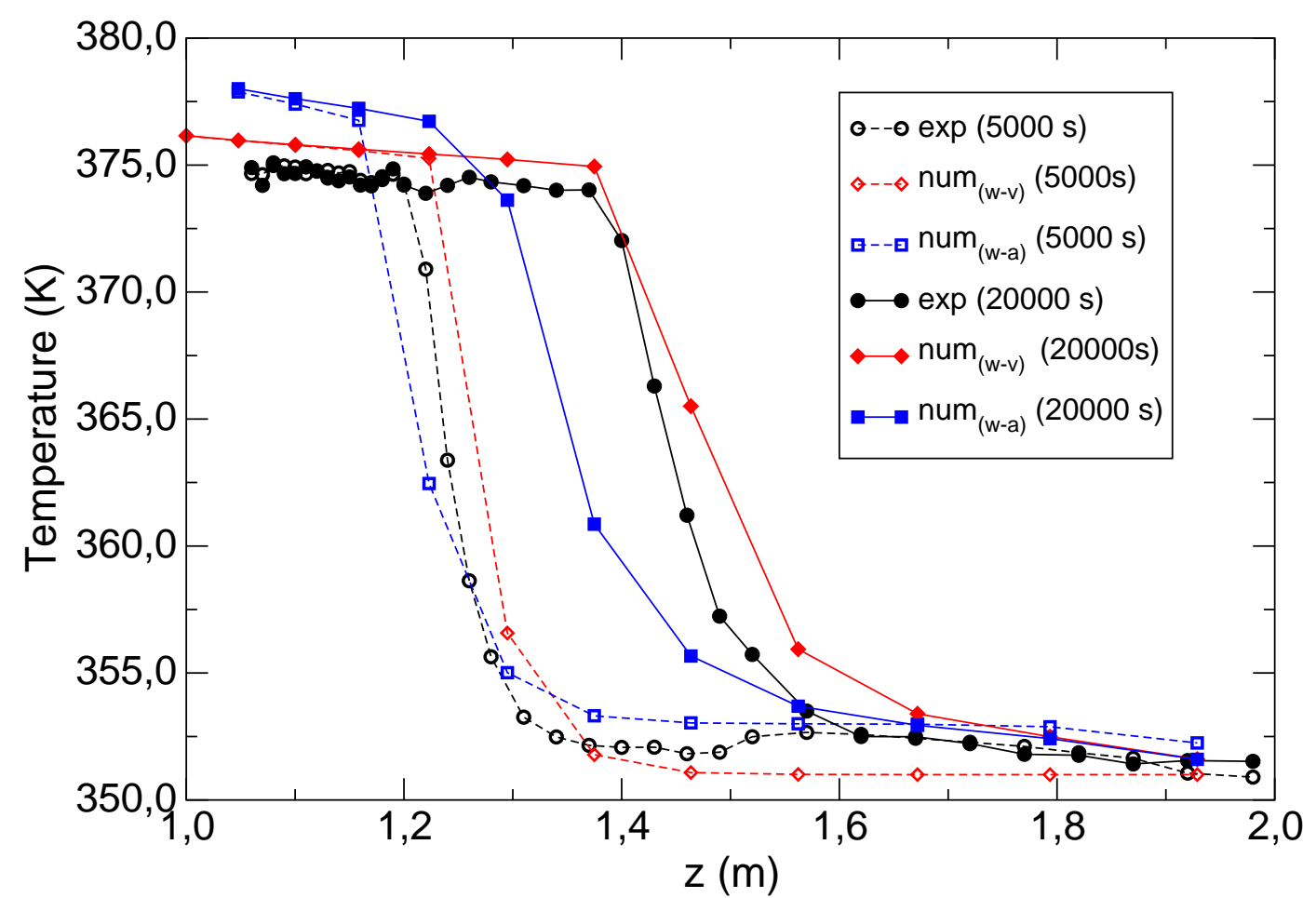

Figure 13. Temperature profiles along axis $z(1 m<z<2 m)$ at $(x, y)=(0,0)$ and at times $t=5000 \mathrm{~s}$ and $t=20000 \mathrm{~s}$. Imposed power $\bar{Q}_{0}=500 \mathrm{~W}$. The diamonds represent numerical results obtained with $P_{c}(S)$ and $k_{r v}(S)$. The squares represent numerical results obtained with $P_{c, w-a}(S)$ and $k_{r v, w-a}(S)$. 


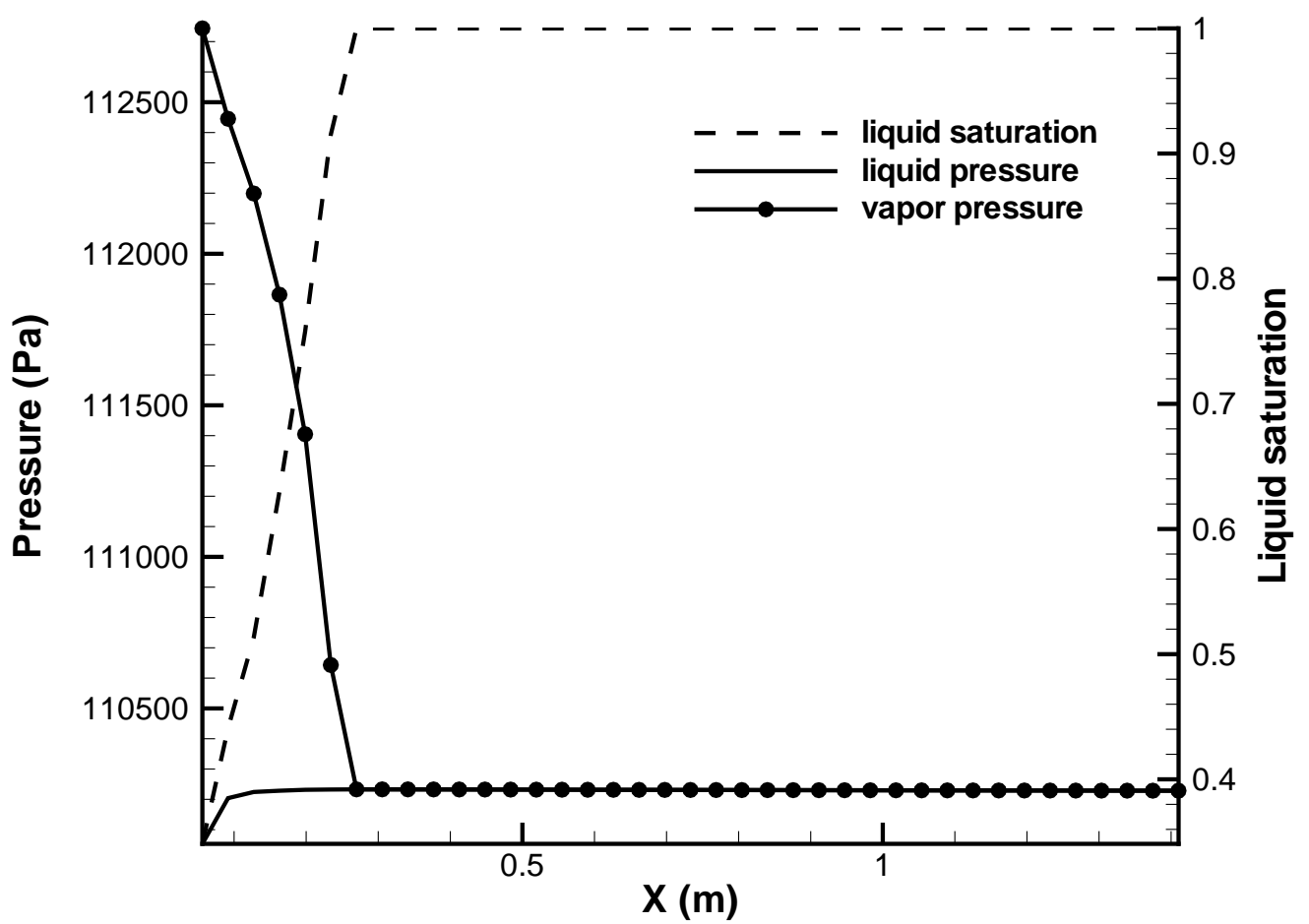

Figure 14. Liquid and vapor pressure and liquid saturation versus $x$ at $t=25000 \mathrm{~s}$. Imposed power: $\bar{Q}_{0}=500 \mathrm{~W}$. 


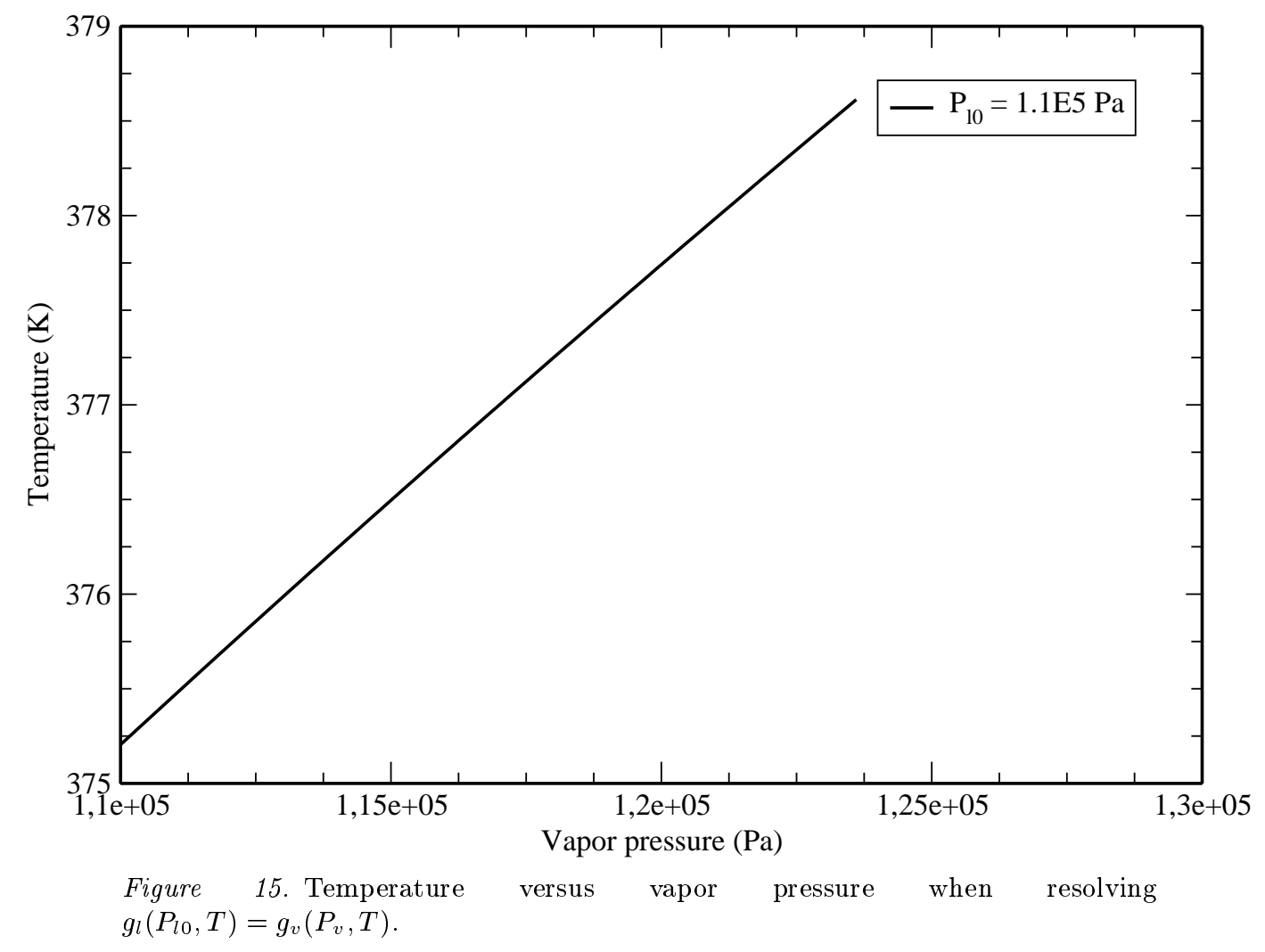




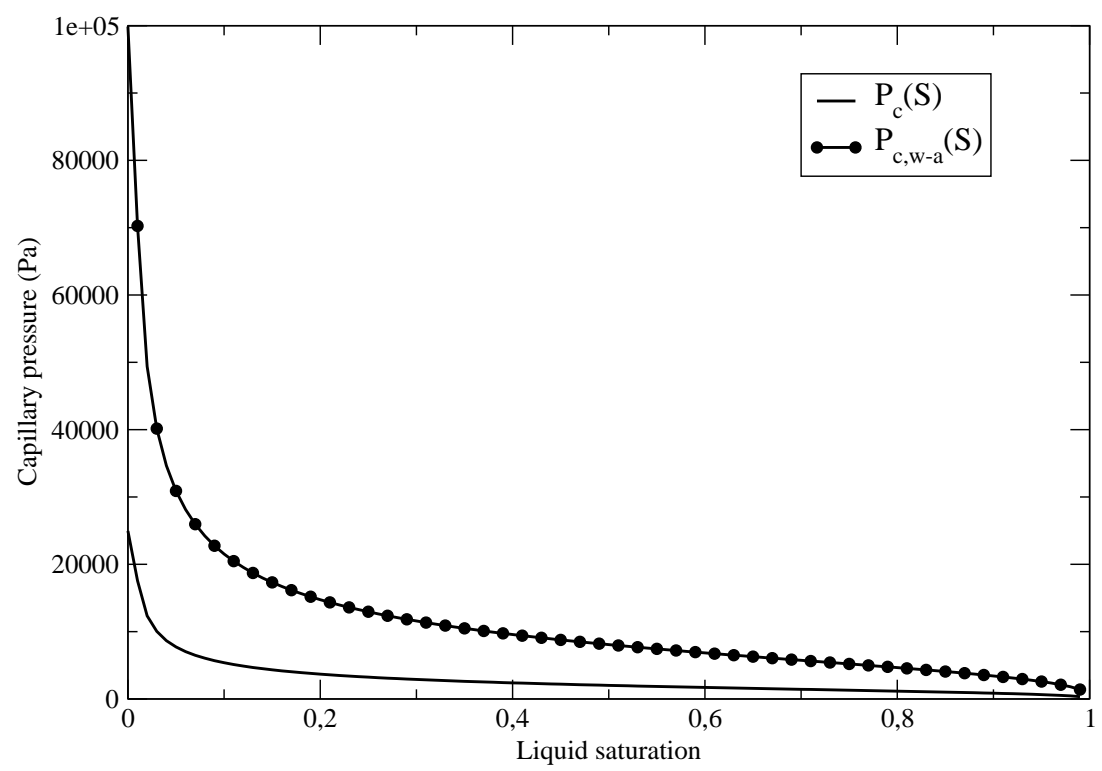

Figure 16. Comparison between the experimentally measured and numerically adapted capillary pressure laws. The circle represents $P_{c, w-a}(S)$ and the solid line represent $P_{c}(S)$ used in the study. 


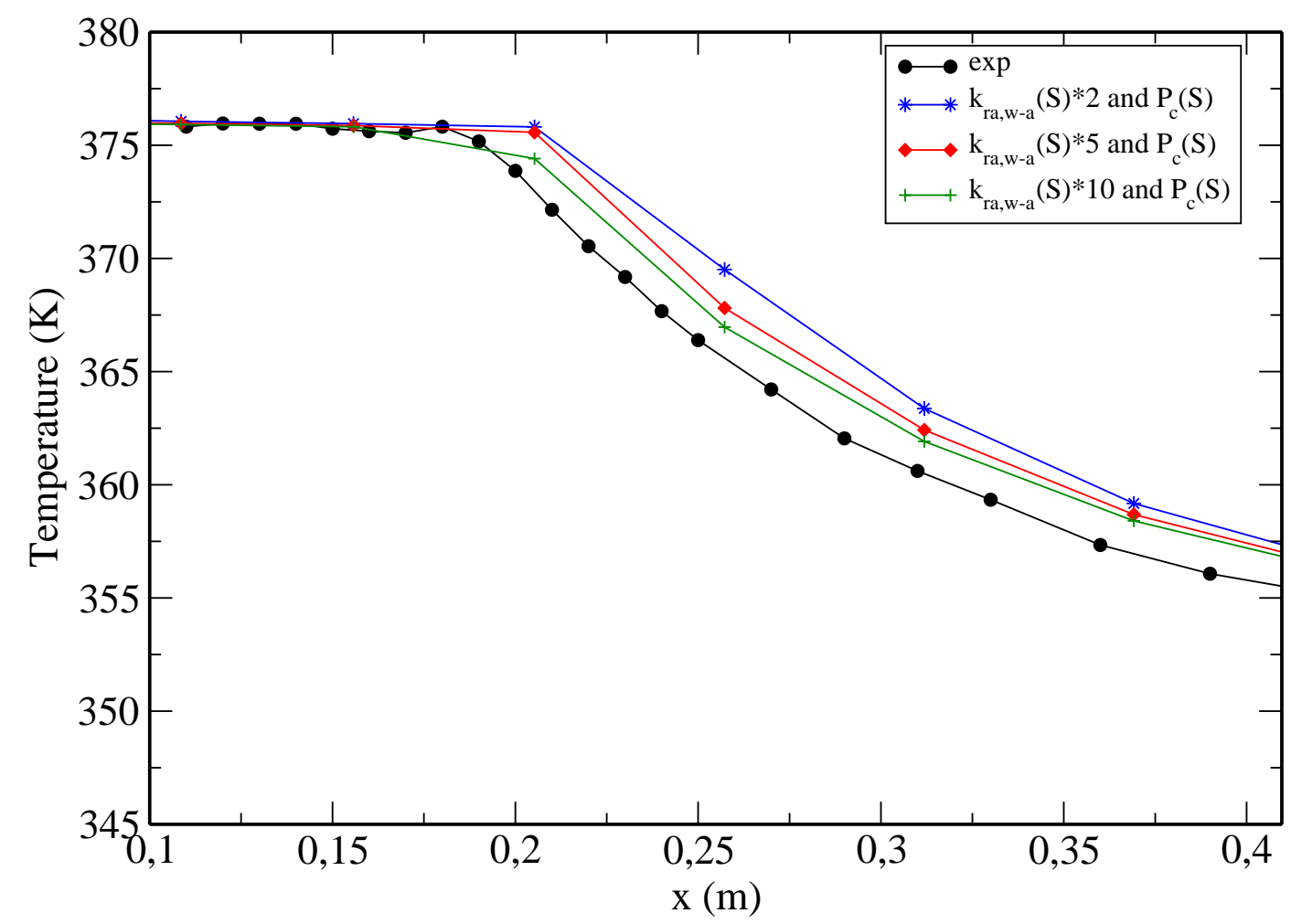

Figure 17. Temperature profiles along axis $x$ at $(y, z)=(0,1)$ at $t=25000 \mathrm{~s}$. Imposed power $\bar{Q}_{0}=500 \mathrm{~W}$. Influence of the vapor permeability. 


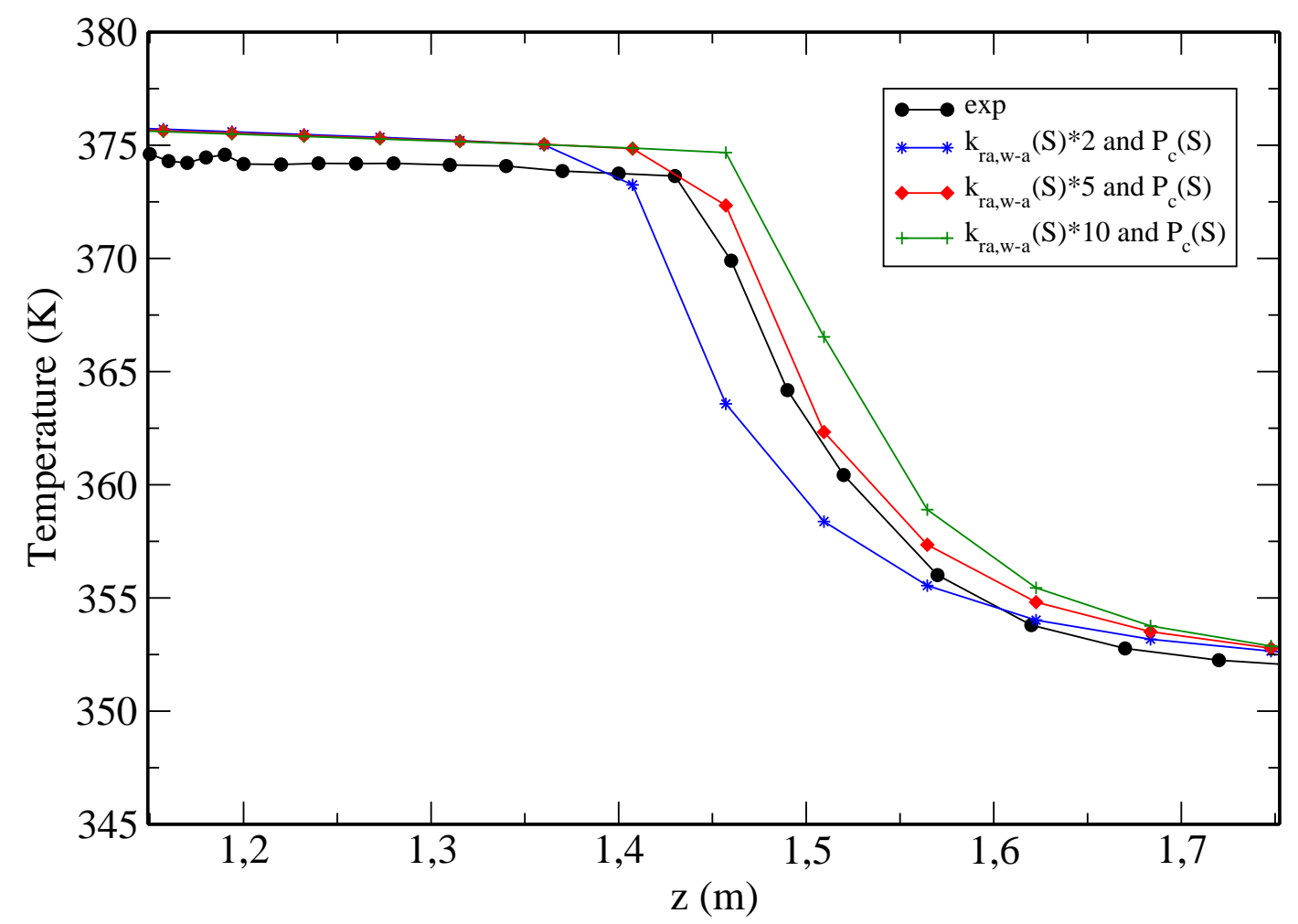

Figure 18. Temperature profiles along axis $z$ at $(x, y)=(0,0)$ at $t=25000 \mathrm{~s}$. Imposed power $\bar{Q}_{0}=500 \mathrm{~W}$. Influence of the vapor permeability. 


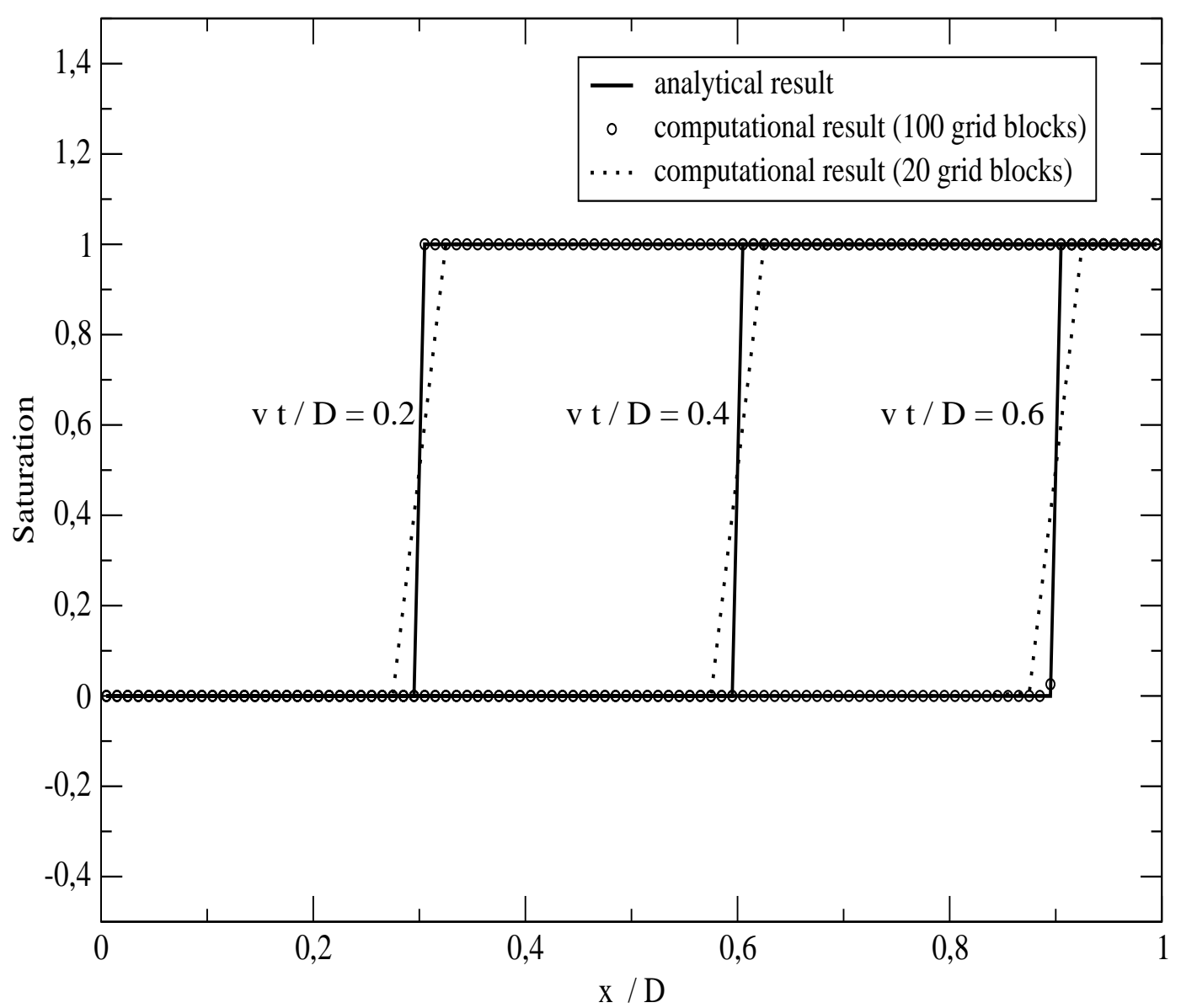

Figure 19. Comparison of the numerical and analytical saturation. $\mathrm{A}=0$. 


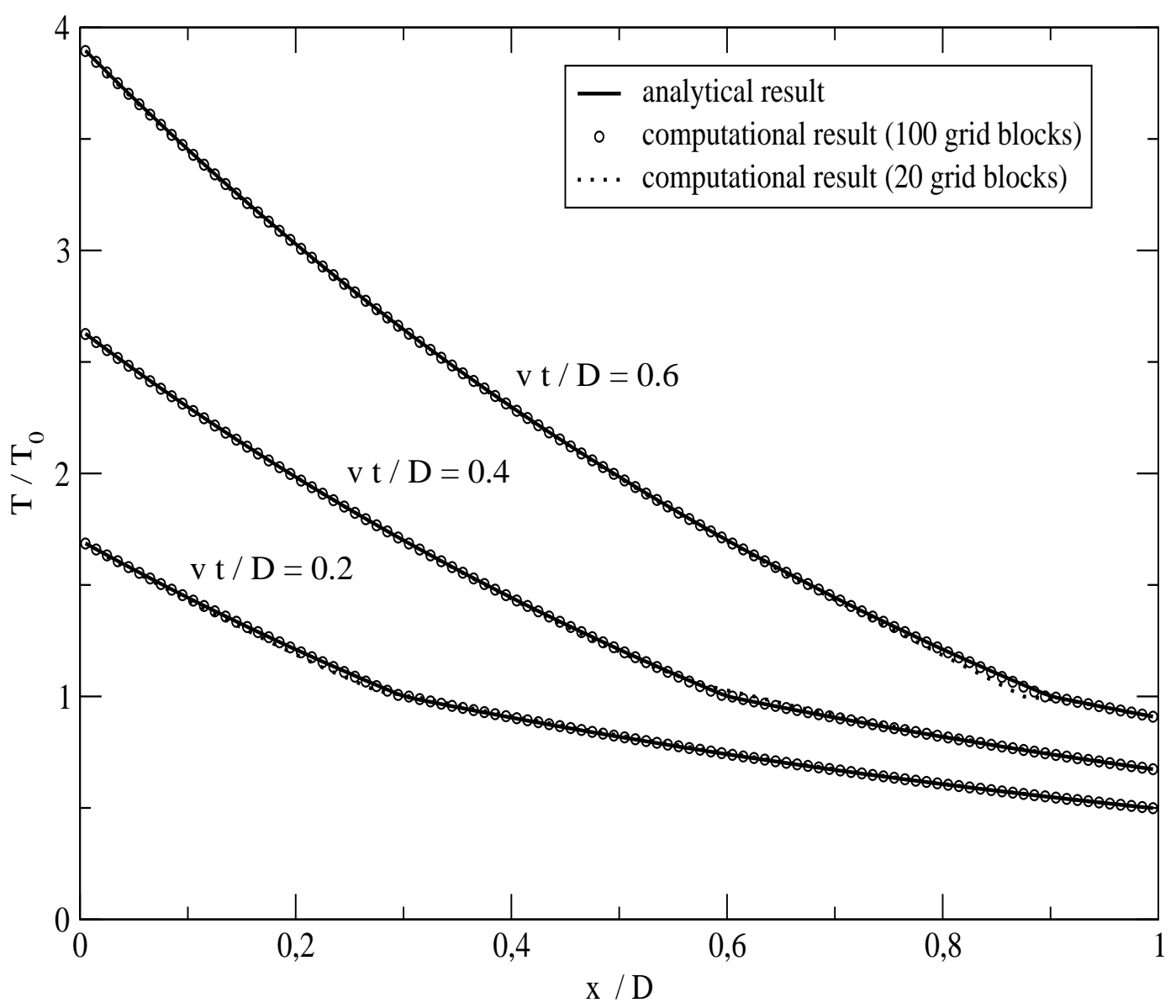

Figure 20. Comparison of the numerical and analytical temperature. $\mathrm{A}=0$. 


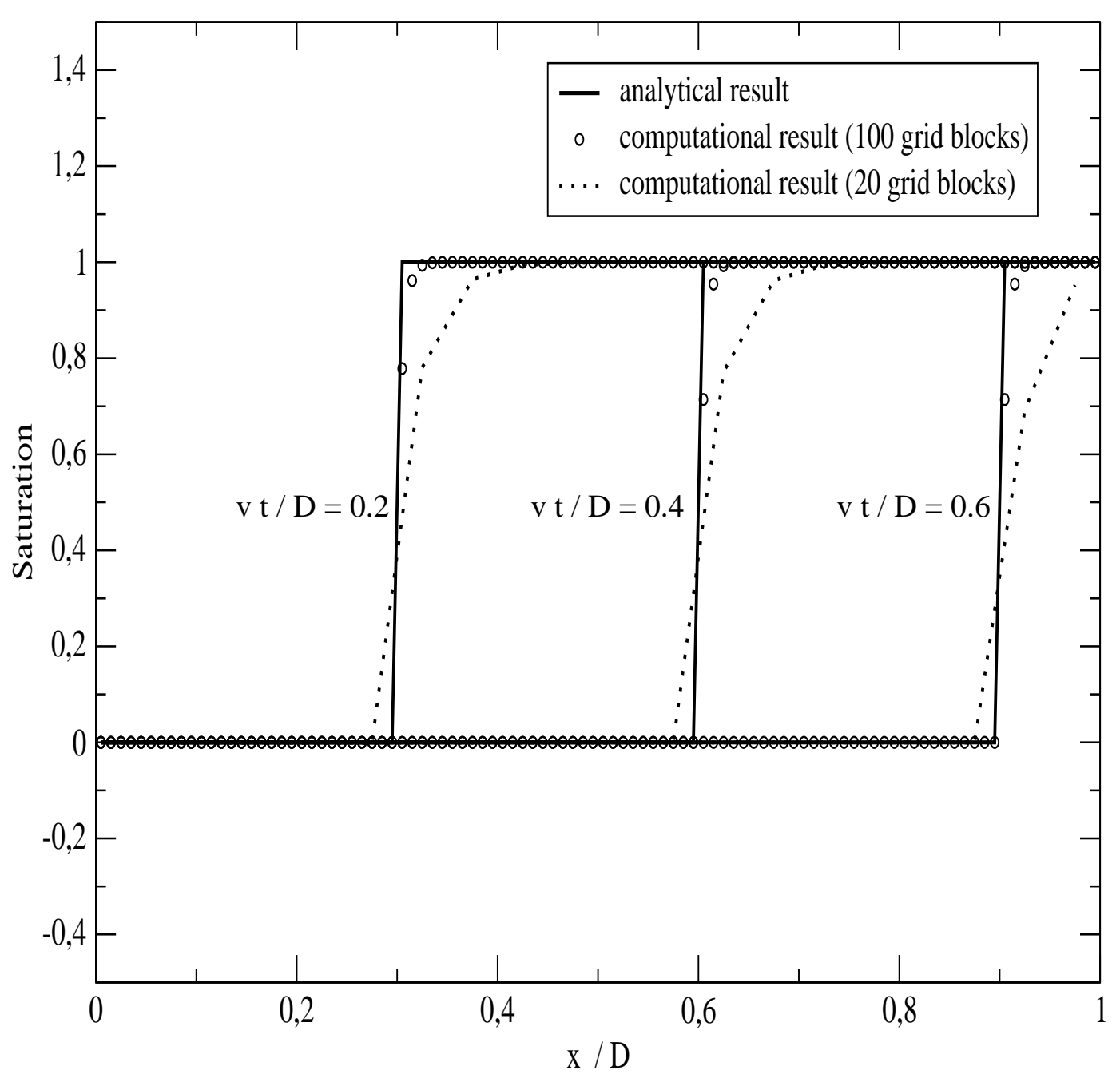

Figure 21. Comparison of the numerical and analytical saturation. $\mathrm{A}=1$. 


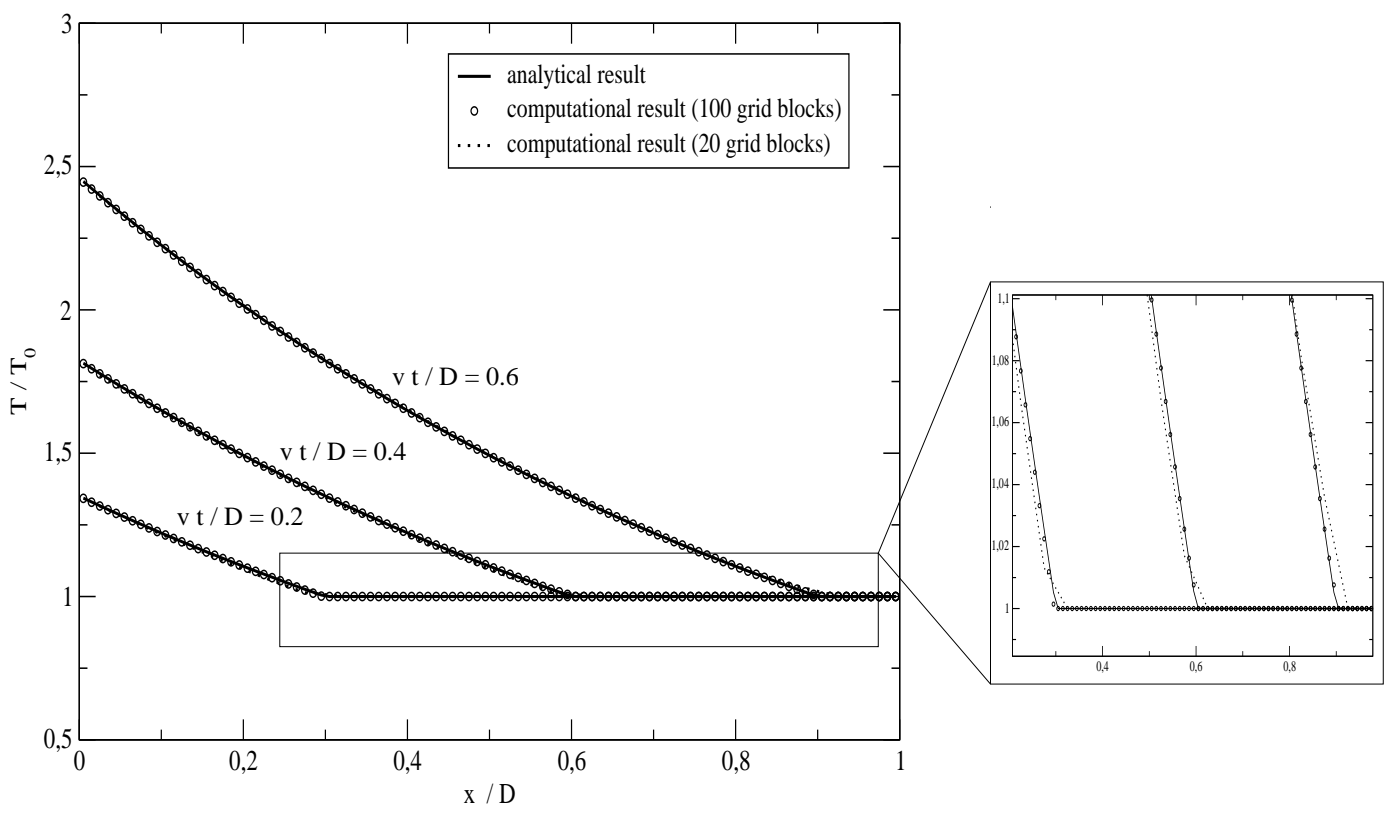

Figure 22. Comparison of the numerical and analytical temperature. $\mathrm{A}=1$. 